\title{
A BEALE-KATO-MAJDA BREAKDOWN CRITERION FOR AN OLDROYD-B FLUID IN THE CREEPING FLOW REGIME*
}

\author{
RAZ KUPFERMAN ${ }^{\dagger}$, CLAUDE MANGOUBI ${ }^{\ddagger}$, AND EDRISS S. TITI ${ }^{\S}$
}

\begin{abstract}
We derive a criterion for the breakdown of solutions to the Oldroyd-B model in $\mathbb{R}^{3}$ in the limit of zero Reynolds number (creeping flow). If the initial stress field is in the Sobolev space $H^{m}\left(\mathbb{R}^{3}\right), m>5 / 2$, then either a unique solution exists within this space indefinitely, or, at the time where the solution breaks down, the time integral of the $L^{\infty}$-norm of the stress tensor must diverge. This result is analogous to the celebrated Beale-Kato-Majda breakdown criterion for the inviscid Euler equations of incompressible fluids.
\end{abstract}

Key words. Oldroyd-B model, local-in-time existence, Beale-Kato-Majda

AMS subject classifications. 35B35, 35Q35

\section{Introduction}

The Oldroyd-B model is a classical model for dilute solutions of polymers suspended in a viscous incompressible solvent [1]. Although it suffers, as a physical model derived from microscopic dynamics, from numerous shortcomings (e.g., polymers are allowed to stretch indefinitely), it is often considered as a prototypical model for viscoelastic fluids, and has therefore been the focus of both analytical and numerical studies.

At present, there is no global-in-time existence theory for the Oldroyd-B model. The notable difference between the Oldroyd-B model and its Newtonian counterpart, the incompressible Navier-Stokes equations, is that in the viscoelastic case, global-intime existence has not even been established in two dimensions nor in the creeping flow regime, i.e., when the momentum equation is a Stokes system. The reason for this difference can be understood by observing structural similarities between the inertialess Oldroyd-B model and the Euler equations (in three dimensions), or the 2D quasi-geostrophic flow equations (in two dimensions) [2].

Since the early 1970s, numerical simulations of the Oldroyd-B model (as well as other viscoelastic models) have been infested by stability and accuracy problems that arise at frustratingly low values of the elasticity parameter (the Weissenberg number) $[3,4]$. While some of these difficulties have been elucidated [5], it is to a large extent still a mystery why computations break down in the low-Reynolds-high-Weissenberg regime. As is often the case in such situations, numerical data are by themselves not sufficient to explain the reasons for this breakdown. In the absence of a wellposedness theory, it is not even clear in what spaces solutions have to be sought. Thus, the development of such a theory is of major importance for both theoretical and practical purposes.

This situation is analogous to that of incompressible Newtonian fluids at high Reynolds number, where global-in-time existence has not yet been established. For a

${ }^{*}$ Received: August 14, 2007; accepted (in revised version): December 4, 2007. Communicated by Weinan E.

${ }^{\dagger}$ Institute of Mathematics, The Hebrew University, Jerusalem 91904 Israel.

${ }^{\ddagger}$ Institute of Mathematics, The Hebrew University, Jerusalem 91904 Israel and CERMICS, École Nationale des Ponts et Chaussées, 77455 Marne la Vallée, France.

$\S$ Faculty of Mathematics and Computer Science, The Weizmann Institute of Science, Rehovot 76100 Israel and Department of Mathematics and Department of Mechanical and Aerospace Engineering, University of California, Irvine CA 92697-3875. 
Newtonian fluid (in three dimensions), however, there is a prominent observation due to Beale, Kato and Majda (BKM) [6], which states a necessary and sufficient condition for the breakdown of solutions in finite time. Specifically, the Euler equations of incompressible inviscid fluids in vorticity formulation take the form

$$
\frac{\partial \boldsymbol{\omega}}{\partial t}+(\boldsymbol{u} \cdot \boldsymbol{\nabla}) \boldsymbol{\omega}=(\boldsymbol{\nabla} \boldsymbol{u})^{T} \boldsymbol{\omega},
$$

with initial condition $\boldsymbol{\omega}(\cdot, 0)=\boldsymbol{\omega}_{0}$. Here $\boldsymbol{\omega}=\nabla \times \boldsymbol{u}$ is the vorticity and $\boldsymbol{u}$ is the velocity field. The BKM theorem states that if $\boldsymbol{\omega}_{0}$ belongs to the Sobolev space $H^{m}\left(\mathbb{R}^{3}\right)$, $m>\frac{3}{2}$, then either there exists a solution $\boldsymbol{\omega}(\cdot, t) \in H^{m}\left(\mathbb{R}^{3}\right)$ for all times, or, if $T^{*}$ is the maximal time of existence of a solution in $H^{m}\left(\mathbb{R}^{3}\right)$, then

$$
\lim _{t \nearrow T^{*}} \int_{0}^{t}\|\boldsymbol{\omega}(\cdot, s)\|_{L^{\infty}} d s=\infty
$$

and in particular,

$$
\limsup _{t \nearrow T^{*}}\|\boldsymbol{\omega}(\cdot, t)\|_{L^{\infty}}=\infty .
$$

That is, the breakup of solutions in any Sobolev norm necessitates the divergence of the $L^{\infty}$-norm of the vorticity. The practical implication of this theorem is that breakdown cannot be attributed, say, to the failure of some high derivative. The blowup of the vorticity itself, in the supremum norm, is the signature of any finitetime breakdown. For another criterion of singularity formation see [7]; for up-to-date surveys see $[8,9]$.

The goal of this paper is to establish a similar result for the three-dimensional Oldroyd-B model, in the zero-Reynolds-number regime. In this regime, a closed equation can be written for the polymeric stress field $\boldsymbol{\sigma}=\boldsymbol{\sigma}(\boldsymbol{x}, t)$; this equation is similar to the vorticity equation (1.1). We start by establishing the local-in-time existence of solutions in any Sobolev space $H^{m}\left(\mathbb{R}^{3}\right), m>5 / 2$. Following then the approach of BKM, we prove that if the initial stress is in $H^{m}\left(\mathbb{R}^{3}\right)$, then either a solution exists for all time, or, if $T^{*}$ is the maximum existence time, then

$$
\lim _{t \nearrow T^{*}} \int_{0}^{t}\|\boldsymbol{\sigma}(\cdot, s)\|_{L^{\infty}} d s=\infty
$$

This result is independent of the Weissenberg number, and in fact holds even if one sets the Weissenberg number to be infinite. From a theoretical point of view, this breakdown condition implies that global-in-time well-posedness hinges on an a priori bound for the supremum norm of the stress.

Recent work along these lines comprises a BKM-type analysis by Chemin and Masmoudi [10]. The notable difference between their analysis and the present work is that they treat the Oldroyd-B model including inertia; however, their analysis is restricted to two-dimensional flows. Lin et al. [11] analyze the inertial Oldroyd$\mathrm{B}$ model without the relaxation term (infinite Weissenberg number) and establish global-in-time existence for small initial data. Finally, Constantin [12] studies a class of kinetic models in the form of a Stokes equation coupled to a nonlinear FokkerPlanck equation, for which he proves global-in-time existence. The extension to a two-dimensional Navier-Stokes system coupled to a nonlinear Fokker-Planck equation appears in Constantin et al. [13] and Constantin and Masmoudi [14]. In both cases the analysis benefits from the fact that the polymeric stress remains bounded by construction, which as our analysis shows, is the key to well-posedness. 


\section{The model}

The Oldroyd-B model describes a fluid in which polymer molecules are suspended in a viscous incompressible solvent. The equations of motion in the creeping flow regime are

$$
\begin{aligned}
0 & =-\boldsymbol{\nabla} p+\nu_{s} \Delta \boldsymbol{u}+\operatorname{div} \boldsymbol{\sigma}, \\
\operatorname{div} \boldsymbol{u} & =0, \\
\frac{\partial \boldsymbol{\sigma}}{\partial t}+(\boldsymbol{u} \cdot \boldsymbol{\nabla}) \boldsymbol{\sigma}-(\boldsymbol{\nabla} \boldsymbol{u})^{T} \boldsymbol{\sigma}-\boldsymbol{\sigma}(\boldsymbol{\nabla} \boldsymbol{u}) & =-\frac{1}{\lambda} \boldsymbol{\sigma}+\frac{\nu_{p}}{\lambda}\left[\boldsymbol{\nabla} \boldsymbol{u}+(\boldsymbol{\nabla} \boldsymbol{u})^{T}\right],
\end{aligned}
$$

where $\boldsymbol{u}$ is the velocity of the fluid, $p$ is the hydrostatic pressure, $\boldsymbol{\sigma}$ is the extra-stress tensor due to the polymer molecules, $\nu_{s}$ is the solvent viscosity, $\nu_{p}$ is the polymeric viscosity, and $\lambda$ is the elastic relaxation time. The velocity gradient is defined with components $(\boldsymbol{\nabla u})_{i j}=\partial u_{j} / \partial x_{i}$.

The first two equations in (2.1) are a Stokes system for an incompressible fluid, whereas the third equation is the Maxwell constitutive equation for the extra-stress [1]. The flow is assumed to take place in the unbounded three-dimensional space $\mathbb{R}^{3}$. Initial data need only be prescribed for the stress, $\boldsymbol{\sigma}(\boldsymbol{x}, 0)=\boldsymbol{\sigma}_{0}(\boldsymbol{x})$.

The system (2.1) can be turned into a closed equation for $\boldsymbol{\sigma}$, by solving the Stokes system and expressing the flow field in terms of the stress field. Specifically, the induced velocity field is given by

$$
u_{j}(\boldsymbol{x})=\frac{1}{8 \pi \nu_{s}} \int_{\mathbb{R}^{3}} M_{j l}^{(0)}(\boldsymbol{y}) \partial_{k} \sigma_{k l}(\boldsymbol{x}-\boldsymbol{y}) d \boldsymbol{y}
$$

where

$$
M_{j l}^{(0)}(\boldsymbol{y})=-\frac{\delta_{j l}}{|y|}+\frac{y_{j} y_{l}}{|y|^{3}}
$$

is the Stokes kernel (see Galdi [15], pp 189-195). Using integration by parts, it can be rewritten as

$$
u_{j}(\boldsymbol{x})=\frac{1}{8 \pi \nu_{s}} \int_{\mathbb{R}^{3}} M_{j k l}^{(1)}(\boldsymbol{y}) \sigma_{k l}(\boldsymbol{x}-\boldsymbol{y}) d \boldsymbol{y}
$$

where

$$
M_{j k l}^{(1)}(\boldsymbol{y})=-\frac{y_{j} \delta_{k l}}{|y|^{3}}+\frac{3 y_{j} y_{k} y_{l}}{|y|^{5}} .
$$

Here and below we adopt the Einstein summation convention, whereby repeated indexes imply a summation unless otherwise specified. In tensor notation we write (2.2) as

$$
\boldsymbol{u}(\boldsymbol{x})=\frac{1}{8 \pi \nu_{s}} \int_{\mathbb{R}^{3}} \boldsymbol{M}^{(1)}(\boldsymbol{y}): \boldsymbol{\sigma}(\boldsymbol{x}-\boldsymbol{y}) d \boldsymbol{y}
$$

where for 2-tensors $\boldsymbol{a}, \boldsymbol{b}$, the : product is defined by $\boldsymbol{a}: \boldsymbol{b}=\operatorname{tr}\left(\boldsymbol{a}^{T} \boldsymbol{b}\right)$. We then rewrite the constitutive equation as a closed evolution equation for the stress field,

$$
\frac{d \boldsymbol{\sigma}}{d t}=\boldsymbol{F}(\boldsymbol{\sigma}), \quad \boldsymbol{\sigma}(\cdot, 0)=\boldsymbol{\sigma}_{0},
$$


where

$$
\boldsymbol{F}(\boldsymbol{\sigma})=-(\boldsymbol{u} \cdot \boldsymbol{\nabla}) \boldsymbol{\sigma}+(\boldsymbol{\nabla} \boldsymbol{u})^{T} \boldsymbol{\sigma}+\boldsymbol{\sigma}(\boldsymbol{\nabla} \boldsymbol{u})-\frac{1}{\lambda} \boldsymbol{\sigma}+\frac{\nu_{p}}{\lambda}\left[\boldsymbol{\nabla} \boldsymbol{u}+(\boldsymbol{\nabla} \boldsymbol{u})^{T}\right]
$$

and $\boldsymbol{u}$ is given by (2.3). Equation (2.4) is viewed as an evolution equation or an ordinary differential equation $(\mathrm{ODE})$ in an infinite-dimensional function space. We observe that the solution $\boldsymbol{\sigma}$ is a symmetric tensor whenever $\boldsymbol{\sigma}_{0}$ is symmetric.

For later use, we derive the linear relation between the stress field $\boldsymbol{\sigma}$ and the velocity gradient $\boldsymbol{\nabla} \boldsymbol{u}$, obtained by differentiating (2.3) and integrating by parts. This yields a singular integral (the integrand is a homogeneous function of degree -3 that averages to zero on the unit sphere), from which one has to extract the singular part,

$$
\partial_{i} u_{j}(\boldsymbol{x})=-\frac{1}{5 \nu_{s}}\left(\sigma_{i j}(\boldsymbol{x})-\frac{1}{3} \delta_{i j} \operatorname{tr} \sigma(\boldsymbol{x})\right)+\frac{1}{8 \pi \nu_{s}}(\mathrm{P} . \mathrm{V} .) \int_{\mathbb{R}^{3}} M_{i j k l}^{(2)}(\boldsymbol{y}) \sigma_{k l}(\boldsymbol{x}-\boldsymbol{y}) d \boldsymbol{y},
$$

where

$$
M_{i j k l}^{(2)}(\boldsymbol{y})=\frac{\delta_{i j} \delta_{k l}}{|y|^{3}}-3 \frac{y_{i} y_{j} \delta_{k l}+2 y_{j} y_{l} \delta_{k i}+\delta_{i j} y_{k} y_{l}}{|y|^{5}}+\frac{15 y_{i} y_{j} y_{k} y_{l}}{|y|^{7}}
$$

and (P.V.) stands for the principal value of a singular integral. In tensor notation,

$$
\boldsymbol{\nabla} \boldsymbol{u}(\boldsymbol{x})=-\frac{1}{5 \nu_{s}}\left(\boldsymbol{\sigma}(\boldsymbol{x})-\frac{\boldsymbol{I}}{3} \operatorname{tr} \boldsymbol{\sigma}(\boldsymbol{x})\right)+\frac{1}{8 \pi \nu_{s}}(\mathrm{P} . \mathrm{V} .) \int_{\mathbb{R}^{3}} \boldsymbol{M}^{(2)}(\boldsymbol{y}): \boldsymbol{\sigma}(\boldsymbol{x}-\boldsymbol{y}) d \boldsymbol{y}
$$

\section{Local-in-time existence}

In this section we prove the local existence and uniqueness of solutions to the Oldroyd-B model (2.1). The proof is based on energy methods, and closely follows the existence proof for the Euler and Navier-Stokes equations in Majda and Bertozzi [16]. Differences between the two cases are highlighted along the treatment. For other results concerning short-time existence or global existence for small initial data see for example $[17,11,18,19,20,21]$ and references therein.

We denote by $H^{m}\left(\mathbb{R}^{3}\right)$ the Sobolev spaces of scalar, vector and tensor fields in $\mathbb{R}^{3}$. The corresponding norms are denoted by $\|\cdot\|_{m}$ and are defined by

$$
\|f\|_{m}=\left(\sum_{|\alpha| \leq m}\left\|D^{\alpha} f\right\|_{0}^{2}\right)^{1 / 2}
$$

where here $f$ denotes either a scalar, a vector or a tensor field and $\alpha=\left(\alpha_{1}, \alpha_{2}, \alpha_{3}\right)$ is a multi-index of derivatives; the norm $\|\cdot\|_{0}$ is the $L^{2}\left(\mathbb{R}^{3}\right)$-norm. For example, if $f=\boldsymbol{f}$ is a tensor field with components $f_{i j}$, then

$$
\left\|D^{\alpha} \boldsymbol{f}\right\|_{0}^{2}=\sum_{i, j=1}^{3} \int_{\mathbb{R}^{3}}\left[\frac{\partial^{|\alpha|}}{\partial x_{1}^{\alpha_{1}} \partial x_{2}^{\alpha_{2}} \partial x_{3}^{\alpha_{3}}} f_{i j}(\boldsymbol{x})\right]^{2} d \boldsymbol{x}
$$

We denote by $\|\cdot\|_{L^{q}}$ and $\|\cdot\|_{L^{\infty}}$ the $L^{q}\left(\mathbb{R}^{3}\right)$ and $L^{\infty}\left(\mathbb{R}^{3}\right)$ norms, respectively. Throughout this section and the next one, we use the symbols $C, K$, to denote either positive constants, or, depending on the context, bounded functions of their arguments.

Our local-in-time existence theorem is: 
Theorem 3.1. Let $\sigma_{0} \in H^{m}\left(\mathbb{R}^{3}\right)$ for $m>5 / 2$. Then there exists a time $T>0$ depending on $\left\|\boldsymbol{\sigma}_{0}\right\|_{m}$ only, so that the Oldroyd-B system (2.1), or equivalently the Hilbert space-valued ODE (2.4), has a solution $\boldsymbol{\sigma}$ in the class

$$
\boldsymbol{\sigma} \in C\left([0, T] ; H^{m}\left(\mathbb{R}^{3}\right)\right) \cap C^{1}\left([0, T] ; H^{m-1}\left(\mathbb{R}^{3}\right)\right) .
$$

Observe that system (2.1) is time-reversible, hence Theorem 3.1 is also valid backward in time.

Since the proof is long and technical, we describe here its outline, and prove each step in a separate subsection. Standard definitions and inequalities are grouped in Appendix A.

Subsec. 3.1. We start by constructing smooth approximations to $\boldsymbol{\sigma}$. We consider a mollified version of $(2.4)$,

$$
\frac{d \boldsymbol{\sigma}_{\varepsilon}}{d t}=\boldsymbol{F}_{\varepsilon}\left(\boldsymbol{\sigma}_{\varepsilon}\right), \quad \boldsymbol{\sigma}_{\varepsilon}(\cdot, 0)=\boldsymbol{\sigma}_{0},
$$

where $\varepsilon>0$ is the mollification parameter,

$$
\begin{aligned}
\boldsymbol{F}_{\varepsilon}\left(\boldsymbol{\sigma}_{\varepsilon}\right)= & -J_{\varepsilon}\left[\boldsymbol{u}_{\varepsilon} \cdot \boldsymbol{\nabla}\left(J_{\varepsilon} \boldsymbol{\sigma}_{\varepsilon}\right)\right]+\left(\boldsymbol{\nabla} \boldsymbol{u}_{\varepsilon}\right)^{T} \boldsymbol{\sigma}_{\varepsilon}+\boldsymbol{\sigma}_{\varepsilon} \boldsymbol{\nabla}\left(\boldsymbol{u}_{\varepsilon}\right) \\
& -\frac{1}{\lambda} \boldsymbol{\sigma}_{\varepsilon}+\frac{\nu_{p}}{\lambda}\left[\boldsymbol{\nabla} \boldsymbol{u}_{\varepsilon}+\left(\boldsymbol{\nabla} \boldsymbol{u}_{\varepsilon}\right)^{T}\right],
\end{aligned}
$$

$\boldsymbol{u}_{\varepsilon}$ is given by the integral (2.3) with $\boldsymbol{\sigma}$ replaced by $\boldsymbol{\sigma}_{\varepsilon}$, and the mollification operator $J_{\varepsilon}$ is defined by (A.1). We then prove

Proposition 3.2. Let $\boldsymbol{\sigma}_{0} \in H^{m}\left(\mathbb{R}^{3}\right)$ for $m>3 / 2$. Then there exists a time $T_{\varepsilon}>0$ depending on $\left\|\boldsymbol{\sigma}_{0}\right\|_{m}$ only, so that (3.2) has a unique solution

$$
\boldsymbol{\sigma}_{\varepsilon} \in C^{1}\left(\left[0, T_{\varepsilon}\right) ; H^{m}\left(\mathbb{R}^{3}\right)\right) .
$$

The main reason for introducing the mollified equation is to enable us to use the classical theory of evolution equations (ODEs) in Banach spaces to prove short time existence of unique solutions. Most importantly, the solutions of the mollified equation are regular enough, which enables us to use classical tools for deriving estimates without a need for additional justification.

Finally, we also observe that by the uniqueness of the solution of (3.2), the tensor $\boldsymbol{\sigma}_{\varepsilon}$ is symmetric whenever $\boldsymbol{\sigma}_{0}$ is symmetric. We will be using this fact later in our estimates.

Subsec. 3.2. Using the continuation theorem for autonomous ODEs, and an a priori estimate of the form

$$
\sup _{0 \leq t \leq T}\left\|\boldsymbol{\sigma}_{\varepsilon}\right\|_{m} \leq K\left(\left\|\boldsymbol{\sigma}_{0}\right\|_{m}, T\right),
$$

where $T=T\left(\left\|\sigma_{0}\right\|_{m}\right)$ is independent of $\varepsilon$, we show that the family of mollified solutions $\boldsymbol{\sigma}_{\varepsilon}$ can be continued uniformly up to a common time $T$. Here we need a slightly higher degree of regularity:

Proposition 3.3. Let $\boldsymbol{\sigma}_{0} \in H^{m}\left(\mathbb{R}^{3}\right)$ for $m>5 / 2$. Then there exists a time $T=$ $T\left(\left\|\boldsymbol{\sigma}_{0}\right\|_{m}\right)>0$ independent of $\varepsilon$, such that the mollified equation (3.2) has a unique solution $\boldsymbol{\sigma}_{\varepsilon} \in C^{1}\left([0, T], H^{m}\left(\mathbb{R}^{3}\right)\right)$, satisfying the uniform bound (3.3). 
Subsec. 3.3. We show that the family of mollified solutions, $\boldsymbol{\sigma}_{\varepsilon}$, forms a Cauchy sequence in $C\left([0, T], L^{2}\left(\mathbb{R}^{3}\right)\right)$, hence strongly converges to a function $\boldsymbol{\sigma} \in C\left([0, T], L^{2}\left(\mathbb{R}^{3}\right)\right)$.

Proposition 3.4. Let $\boldsymbol{\sigma}_{0} \in H^{m}\left(\mathbb{R}^{3}\right)$ for $m>5 / 2$. Then the family of mollified solutions $\boldsymbol{\sigma}_{\varepsilon} \in C^{1}\left([0, T], H^{m}\left(\mathbb{R}^{3}\right)\right)$ forms, as $\varepsilon \rightarrow 0$, a Cauchy sequence in $C\left([0, T], L^{2}\left(\mathbb{R}^{3}\right)\right)$, hence converges to a function, which we denote by $\boldsymbol{\sigma}$. Moreover, for every $0 \leq t \leq T$, we have $\boldsymbol{\sigma}(\cdot, t) \in H^{m}\left(\mathbb{R}^{3}\right)$, and $\boldsymbol{\sigma}$ satisfies the same bound,

$$
\sup _{0 \leq t \leq T}\|\boldsymbol{\sigma}\|_{m} \leq K\left(\left\|\boldsymbol{\sigma}_{0}\right\|_{m}, T\right),
$$

as the family of mollified solutions.

Subsec. 3.4. Using the technique of interpolation we show that $\boldsymbol{\sigma}_{\varepsilon}$ strongly converges to $\boldsymbol{\sigma}$ in all intermediate norms $C\left([0, T], H^{m^{\prime}}\left(\mathbb{R}^{3}\right)\right), 0<m^{\prime}<m$. We then proceed to prove continuity of the limit in the highest norm, $\boldsymbol{\sigma} \in C\left([0, T], H^{m}\left(\mathbb{R}^{3}\right)\right)$.

Proposition 3.5. Let $\boldsymbol{\sigma}_{0} \in H^{m}\left(\mathbb{R}^{3}\right)$ for $m>5 / 2$. Then the $C\left([0, T], H^{m^{\prime}}\left(\mathbb{R}^{3}\right)\right)$ limit $\boldsymbol{\sigma}$ of $\boldsymbol{\sigma}_{\varepsilon}$, for every $m^{\prime} \in(0, m)$, is continuous in $\boldsymbol{\sigma} \in C\left([0, T], H^{m}\left(\mathbb{R}^{3}\right)\right)$.

Subsec. 3.5. We finally show that $\boldsymbol{\sigma}$, the limit of $\boldsymbol{\sigma}_{\varepsilon}$, is a solution of (2.4) in the space

$$
\boldsymbol{\sigma} \in C\left([0, T], H^{m}\left(\mathbb{R}^{3}\right)\right) \cap C^{1}\left([0, T], H^{m-1}\left(\mathbb{R}^{3}\right)\right) .
$$

3.1. Local-in-time existence of mollified solutions. In this subsection we prove Proposition 3.2. We approximate the Oldroyd-B system (2.1), or equivalently, the Hilbert space-valued ODE (evolution equation) (2.4) by a mollified equation for a smooth approximation $\boldsymbol{\sigma}_{\varepsilon}$ of $\boldsymbol{\sigma}$,

$$
\frac{d \boldsymbol{\sigma}_{\varepsilon}}{d t}=\boldsymbol{F}_{\varepsilon}\left(\boldsymbol{\sigma}_{\varepsilon}\right), \quad \boldsymbol{\sigma}_{\varepsilon}(\cdot, 0)=\boldsymbol{\sigma}_{0},
$$

where

$$
\begin{aligned}
\boldsymbol{F}_{\varepsilon}\left(\boldsymbol{\sigma}_{\varepsilon}\right)= & -J_{\varepsilon}\left[\boldsymbol{u}_{\varepsilon} \cdot \boldsymbol{\nabla}\left(J_{\varepsilon} \boldsymbol{\sigma}_{\varepsilon}\right)\right]+\left(\boldsymbol{\nabla} \boldsymbol{u}_{\varepsilon}\right)^{T} \boldsymbol{\sigma}_{\varepsilon}+\boldsymbol{\sigma}_{\varepsilon}\left(\boldsymbol{\nabla} \boldsymbol{u}_{\varepsilon}\right) \\
& -\frac{1}{\lambda} \boldsymbol{\sigma}_{\varepsilon}+\frac{\nu_{p}}{\lambda}\left[\boldsymbol{\nabla} \boldsymbol{u}_{\varepsilon}+\left(\boldsymbol{\nabla} \boldsymbol{u}_{\varepsilon}\right)^{T}\right] ;
\end{aligned}
$$

$\boldsymbol{u}_{\varepsilon}$ is given by (2.3) with $\boldsymbol{\sigma}$ replaced by $\boldsymbol{\sigma}_{\varepsilon}$, and the mollification operator $J_{\varepsilon}$ is defined by (A.1) in the appendix. Comparing with (2.5), we note that mollification is only used in the advection term. As will be shown, the gradient of $\boldsymbol{u}_{\varepsilon}$ has the same degree of regularity, with respect to the $H^{m}\left(\mathbb{R}^{3}\right)$-norms, as $\boldsymbol{\sigma}_{\varepsilon}$, hence no additional mollification is needed.

To prove that (3.4) has a local-in-time solution we use Picard's theorem over Banach spaces. Specifically, we work within the Banach space $H^{m}\left(\mathbb{R}^{3}\right)$ with $m>3 / 2$. Picard's theorem for functional evolution differential equations states that if there exists an open subset $O \subset H^{m}\left(\mathbb{R}^{3}\right)$ such that

1. $\boldsymbol{F}_{\varepsilon}: O \rightarrow H^{m}\left(\mathbb{R}^{3}\right)$, and

2. $\boldsymbol{F}_{\varepsilon}$ is locally Lipschitz continuous, i.e., for any $\boldsymbol{\sigma} \in O$ there exists an open neighborhood of $\boldsymbol{\sigma}, U \subset O$, and a constant $L>0$ such that for every $\boldsymbol{\tau}_{1}, \boldsymbol{\tau}_{2} \in U$

$$
\left\|\boldsymbol{F}_{\varepsilon}\left(\boldsymbol{\tau}_{1}\right)-\boldsymbol{F}_{\varepsilon}\left(\boldsymbol{\tau}_{2}\right)\right\|_{m} \leq L\left\|\boldsymbol{\tau}_{1}-\boldsymbol{\tau}_{2}\right\|_{m},
$$


then there exists, for every $\boldsymbol{\sigma}_{0} \in O$, a time $T_{\varepsilon}>0$ and a unique solution $\boldsymbol{\sigma}_{\varepsilon} \in$ $C^{1}\left(\left[0, T_{\varepsilon}\right) ; O\right)$ of $(3.4)$.

Two properties that are being used extensively throughout this section are:

(i) the Calderón-Zygmund (CZ) inequality (A.11) (see Appendix), from which it follows that $\boldsymbol{\sigma}_{\varepsilon} \in H^{m}\left(\mathbb{R}^{3}\right)$ implies that the velocity gradient $\boldsymbol{\nabla} \boldsymbol{u}_{\varepsilon}$, and a fortiori the velocity $\boldsymbol{u}_{\varepsilon}$ itself, are in $H^{m}\left(\mathbb{R}^{3}\right)$ as well,

$$
\left\|\boldsymbol{u}_{\varepsilon}\right\|_{m} \leq \frac{c_{m}}{\nu_{s}}\left\|\boldsymbol{\sigma}_{\varepsilon}\right\|_{m}, \quad\left\|\nabla \boldsymbol{u}_{\varepsilon}\right\|_{m} \leq \frac{\tilde{c}_{m}}{\nu_{s}}\left\|\boldsymbol{\sigma}_{\varepsilon}\right\|_{m} .
$$

(ii) For $m>3 / 2, H^{m}\left(\mathbb{R}^{3}\right)$ forms a Banach algebra, i.e.,

$$
\|f g\|_{m} \leq c\|f\|_{m}\|g\|_{m}
$$

Combining these two properties with the smoothing properties (A.3)-(A.4) of $J_{\varepsilon}$ (see Appendix), it follows at once that $\boldsymbol{F}_{\varepsilon}$ is a mapping $H^{m}\left(\mathbb{R}^{3}\right) \rightarrow H^{m}\left(\mathbb{R}^{3}\right)$. We set

$$
O=\left\{\boldsymbol{\sigma} \in H^{m}\left(\mathbb{R}^{3}\right):\|\boldsymbol{\sigma}\|_{m}<r\right\},
$$

where $r$ is sufficiently large such that $\sigma_{0} \in O$. It remains to show that there exists a positive constant $L=L(r)$, such that $\boldsymbol{F}_{\varepsilon}$ is Lipschitz continuous in $O$ with constant $L$.

To avoid lengthy expressions, we split $\boldsymbol{F}_{\varepsilon}$ into a sum of four terms,

$$
\boldsymbol{F}_{\varepsilon}=\boldsymbol{F}_{1}+\boldsymbol{F}_{2}+\boldsymbol{F}_{3}+\boldsymbol{F}_{4},
$$

where

$$
\begin{array}{lllrl}
\boldsymbol{F}_{1}\left(\boldsymbol{\sigma}_{\varepsilon}\right) & =-J_{\varepsilon}\left[\boldsymbol{u}_{\varepsilon} \cdot \boldsymbol{\nabla}\left(J_{\varepsilon} \boldsymbol{\sigma}_{\varepsilon}\right)\right], & & \boldsymbol{F}_{2}\left(\boldsymbol{\sigma}_{\varepsilon}\right)=\left(\boldsymbol{\nabla} \boldsymbol{u}_{\varepsilon}\right)^{T} \boldsymbol{\sigma}_{\varepsilon}+\boldsymbol{\sigma}_{\varepsilon}\left(\boldsymbol{\nabla} \boldsymbol{u}_{\varepsilon}\right), \\
\boldsymbol{F}_{3}\left(\boldsymbol{\sigma}_{\varepsilon}\right)=-\frac{1}{\lambda} \boldsymbol{\sigma}_{\varepsilon}, & \boldsymbol{F}_{4}\left(\boldsymbol{\sigma}_{\varepsilon}\right)=\frac{\nu_{p}}{\lambda}\left[\boldsymbol{\nabla} \boldsymbol{u}_{\varepsilon}+\left(\boldsymbol{\nabla} \boldsymbol{u}_{\varepsilon}\right)^{T}\right],
\end{array}
$$

and show that each of these four terms is Lipschitz continuous. That is, let $\boldsymbol{\tau}_{1}, \boldsymbol{\tau}_{2} \in O$ and let $\boldsymbol{u}_{1}, \boldsymbol{u}_{2}$ be their corresponding velocity fields satisfying the Stokes system (2.3); we show that each of the $\boldsymbol{F}_{j}$ verifies a bound of the type

$$
\left\|\boldsymbol{F}_{j}\left(\boldsymbol{\tau}_{1}\right)-\boldsymbol{F}_{j}\left(\boldsymbol{\tau}_{2}\right)\right\|_{m} \leq L\left(\varepsilon,\left\|\boldsymbol{\tau}_{1}\right\|_{m},\left\|\boldsymbol{\tau}_{2}\right\|_{m}\right)\left\|\boldsymbol{\tau}_{1}-\boldsymbol{\tau}_{2}\right\|_{m},
$$

where $L$ is a monotonic function of its last two arguments, and hence it is bounded by $L(\varepsilon, r, r)$.

The Lipschitz continuity of the linear function $\boldsymbol{F}_{3}$ is trivial. The Lipschitz continuity of $\boldsymbol{F}_{4}$ follows from the CZ inequality (A.11), which implies

$$
\left\|\nabla \boldsymbol{u}_{2}-\nabla \boldsymbol{u}_{1}\right\|_{m} \leq \frac{C}{\nu_{s}}\left\|\boldsymbol{\tau}_{2}-\boldsymbol{\tau}_{1}\right\|_{m}
$$

For the advection term $\boldsymbol{F}_{1}$ we have

$$
\begin{aligned}
\left\|\boldsymbol{F}_{1}\left(\boldsymbol{\tau}_{2}\right)-\boldsymbol{F}_{1}\left(\boldsymbol{\tau}_{1}\right)\right\|_{m} & =\left\|J_{\varepsilon}\left[\boldsymbol{u}_{2} \cdot \boldsymbol{\nabla}\left(J_{\varepsilon} \boldsymbol{\tau}_{2}\right)\right]-J_{\varepsilon}\left[\boldsymbol{u}_{1} \cdot \boldsymbol{\nabla}\left(J_{\varepsilon} \boldsymbol{\tau}_{1}\right)\right]\right\|_{m} \\
& \leq C\left\|\boldsymbol{u}_{2} \cdot \boldsymbol{\nabla}\left(J_{\varepsilon} \boldsymbol{\tau}_{2}\right)-\boldsymbol{u}_{1} \cdot \boldsymbol{\nabla}\left(J_{\varepsilon} \boldsymbol{\tau}_{1}\right)\right\|_{m} \\
& \leq C\left\|\boldsymbol{u}_{2} \cdot \boldsymbol{\nabla}\left(J_{\varepsilon}\left(\boldsymbol{\tau}_{2}-\boldsymbol{\tau}_{1}\right)\right)\right\|_{m}+C\left\|\left(\boldsymbol{u}_{2}-\boldsymbol{u}_{1}\right) \cdot \boldsymbol{\nabla}\left(J_{\varepsilon} \boldsymbol{\tau}_{1}\right)\right\|_{m}
\end{aligned}
$$


where in the passage from the first to the second line we used (A.3) with $k=0$, and in the passage from the second to the third line we added and subtracted equal terms and used the triangle inequality. Using the Sobolev calculus inequality (A.6),

$$
\begin{aligned}
\left\|\boldsymbol{F}_{1}\left(\boldsymbol{\tau}_{2}\right)-\boldsymbol{F}_{1}\left(\boldsymbol{\tau}_{1}\right)\right\|_{m} \leq & C\left[\left\|\boldsymbol{u}_{2}\right\|_{L^{\infty}}\left\|\boldsymbol{\nabla}\left(J_{\varepsilon}\left(\boldsymbol{\tau}_{2}-\boldsymbol{\tau}_{1}\right)\right)\right\|_{m}\right. \\
& +\left\|\boldsymbol{\nabla}\left(J_{\varepsilon}\left(\boldsymbol{\tau}_{2}-\boldsymbol{\tau}_{1}\right)\right)\right\|_{L^{\infty}}\left\|\boldsymbol{u}_{2}\right\|_{m}+\left\|\boldsymbol{u}_{2}-\boldsymbol{u}_{1}\right\|_{L^{\infty}}\left\|\boldsymbol{\nabla}\left(J_{\varepsilon} \boldsymbol{\tau}_{1}\right)\right\|_{m} \\
& \left.+\left\|\boldsymbol{\nabla}\left(J_{\varepsilon} \boldsymbol{\tau}_{1}\right)\right\|_{L^{\infty}}\left\|\boldsymbol{u}_{2}-\boldsymbol{u}_{1}\right\|_{m}\right] .
\end{aligned}
$$

Using then property (A.3) of the mollification operator and the Sobolev embedding (A.9), we obtain:

$$
\left\|\boldsymbol{F}_{1}\left(\boldsymbol{\tau}_{2}\right)-\boldsymbol{F}_{1}\left(\boldsymbol{\tau}_{1}\right)\right\|_{m} \leq \frac{C}{\varepsilon}\left(\left\|\boldsymbol{u}_{2}\right\|_{m}\left\|\boldsymbol{\tau}_{2}-\boldsymbol{\tau}_{1}\right\|_{m}+\left\|\boldsymbol{\tau}_{1}\right\|_{m}\left\|\boldsymbol{u}_{2}-\boldsymbol{u}_{1}\right\|_{m}\right)
$$

Finally, using CZ inequality,

$$
\left\|\boldsymbol{F}_{1}\left(\boldsymbol{\tau}_{2}\right)-\boldsymbol{F}_{1}\left(\boldsymbol{\tau}_{1}\right)\right\|_{m} \leq C\left(\varepsilon,\left\|\boldsymbol{\tau}_{1}\right\|_{m},\left\|\boldsymbol{\tau}_{2}\right\|_{m}\right)\left\|\boldsymbol{\tau}_{2}-\boldsymbol{\tau}_{1}\right\|_{m}
$$

The deformation term $\boldsymbol{F}_{2}$ remains. Using the Banach algebra property (A.5),

$$
\begin{aligned}
\left\|\boldsymbol{F}_{2}\left(\boldsymbol{\tau}_{2}\right)-\boldsymbol{F}_{2}\left(\boldsymbol{\tau}_{1}\right)\right\|_{m} & =\left\|\left(\boldsymbol{\nabla} \boldsymbol{u}_{1}\right)^{T} \boldsymbol{\tau}_{1}+\boldsymbol{\tau}_{1}\left(\boldsymbol{\nabla} \boldsymbol{u}_{1}\right)-\left(\boldsymbol{\nabla} \boldsymbol{u}_{2}\right)^{T} \boldsymbol{\tau}_{2}-\boldsymbol{\tau}_{2}\left(\boldsymbol{\nabla} \boldsymbol{u}_{2}\right)\right\|_{m} \\
& \leq 2\left\|\boldsymbol{\tau}_{1}\left(\boldsymbol{\nabla} \boldsymbol{u}_{1}\right)-\boldsymbol{\tau}_{2}\left(\boldsymbol{\nabla} \boldsymbol{u}_{2}\right)\right\|_{m} \\
& \leq 2\left\|\boldsymbol{\nabla} \boldsymbol{u}_{1}\right\|_{m}\left\|\boldsymbol{\tau}_{2}-\boldsymbol{\tau}_{1}\right\|_{m}+2\left\|\boldsymbol{\tau}_{2}\right\|_{m}\left\|\boldsymbol{\nabla} \boldsymbol{u}_{2}-\boldsymbol{\nabla} \boldsymbol{u}_{1}\right\|_{m}
\end{aligned}
$$

One more application of the $\mathrm{CZ}$ inequality gives,

$$
\left\|\boldsymbol{F}_{2}\left(\boldsymbol{\tau}_{2}\right)-\boldsymbol{F}_{2}\left(\boldsymbol{\tau}_{1}\right)\right\|_{m} \leq C\left(\left\|\boldsymbol{\tau}_{1}\right\|_{m}+\left\|\boldsymbol{\tau}_{2}\right\|_{m}\right)\left\|\boldsymbol{\tau}_{2}-\boldsymbol{\tau}_{1}\right\|_{m}
$$

This shows that $\boldsymbol{F}_{\varepsilon}$ is locally Lipschitz in $O$, giving local existence for the mollified Equation (3.4).

3.2. Energy estimates for the mollified solutions. In this subsection we prove Proposition 3.3, i.e., that the mollified solutions can be continued uniformly up to a time $T>0$ that does not depend on $\varepsilon$. To do so, we first obtain an a priori estimate, whereby if the solution $\boldsymbol{\sigma}_{\varepsilon}$ exists up to time $T$, then

$$
\sup _{0 \leq t \leq T}\left\|\boldsymbol{\sigma}_{\varepsilon}(\cdot, t)\right\|_{m} \leq C\left(T,\left\|\boldsymbol{\sigma}_{0}\right\|_{m}\right) \equiv K
$$

where all the $\boldsymbol{\sigma}_{\varepsilon}$ have the same initial condition. The existence of the solution up to that time follows from the continuation theorem for autonomous ODEs in Banach spaces. Taking for domain

$$
O=\left\{\boldsymbol{\sigma} \in H^{m}\left(\mathbb{R}^{3}\right):\|\boldsymbol{\sigma}\|_{m} \leq K\right\},
$$

the solution either exists up to time $T$, or leaves the set $O$ before that time, which we would have ruled out by the above estimate.

To obtain an a priori uniform bound on $\left\|\boldsymbol{\sigma}_{\varepsilon}(\cdot, t)\right\|_{m}$, we use an energy estimate. Starting from the mollified equation (3.4), we take its $\alpha$-th derivative $(|\alpha| \leq m)$, and then an inner product with $D^{\alpha} \boldsymbol{\sigma}_{\varepsilon}$. This yields an "energy" equation,

$$
\begin{aligned}
\frac{1}{2} \frac{d}{d t}\left\|D^{\alpha} \boldsymbol{\sigma}_{\varepsilon}\right\|_{0}^{2} & +\frac{1}{\lambda}\left\|D^{\alpha} \boldsymbol{\sigma}_{\varepsilon}\right\|_{0}^{2}=-\left(D^{\alpha} \boldsymbol{\sigma}_{\varepsilon}, D^{\alpha} J_{\varepsilon}\left[\boldsymbol{u}_{\varepsilon} \cdot \nabla\left(J_{\varepsilon} \boldsymbol{\sigma}_{\varepsilon}\right)\right]\right) \\
& +2\left(D^{\alpha} \boldsymbol{\sigma}_{\varepsilon}, D^{\alpha}\left[\boldsymbol{\sigma}_{\varepsilon}\left(\boldsymbol{\nabla} \boldsymbol{u}_{\varepsilon}\right)\right]\right)+\frac{2 \nu_{p}}{\lambda}\left(D^{\alpha} \boldsymbol{\sigma}_{\varepsilon}, D^{\alpha}\left(\boldsymbol{\nabla} \boldsymbol{u}_{\varepsilon}\right)\right) \\
\equiv & I_{1}+I_{2}+I_{3},
\end{aligned}
$$


where we used the symmetry of $\boldsymbol{\sigma}_{\varepsilon}$ in the last two terms. We observe that $\boldsymbol{\sigma}_{\varepsilon}$ is as smooth as the initial data $\boldsymbol{\sigma}_{0} \in H^{m}\left(\mathbb{R}^{3}\right)$, and $J_{\varepsilon} \boldsymbol{\sigma}_{\varepsilon} \in C^{\infty}\left(\mathbb{R}^{3}\right)$; therefore the above estimates should be interpreted in the strong sense. In particular, the time derivative is classical and we do not require additional justification. Since we need a bound that does not depend on $\varepsilon$, we cannot use the smoothing properties of $J_{\varepsilon}$. On the other hand, we are not concerned with finite-time blowup as long as the time horizon is independent of $\varepsilon$. We start with the advection term. Using the fact that $J_{\varepsilon}$ commutes with weak derivatives and is symmetric, we write

$$
-I_{1}=\left(D^{\alpha}\left(J_{\varepsilon} \boldsymbol{\sigma}_{\varepsilon}\right), D^{\alpha}\left[\boldsymbol{u}_{\varepsilon} \cdot \boldsymbol{\nabla}\left(J_{\varepsilon} \boldsymbol{\sigma}_{\varepsilon}\right)\right]\right) .
$$

We then add and subtract from the second argument of the inner product

$$
\boldsymbol{u}_{\varepsilon} \cdot D^{\alpha} \boldsymbol{\nabla}\left(J_{\varepsilon} \boldsymbol{\sigma}_{\varepsilon}\right),
$$

whose inner product with $D^{\alpha}\left(J_{\varepsilon} \boldsymbol{\sigma}_{\varepsilon}\right)$ vanishes due to the incompressibility of the flow, i.e., due to $\boldsymbol{u}_{\varepsilon}$ being divergence-free. Using the Cauchy-Schwarz inequality and (A.3) for $k=0$, we obtain

$$
I_{1} \leq C\left\|D^{\alpha} \boldsymbol{\sigma}_{\varepsilon}\right\|_{0}\left\|D^{\alpha}\left[\boldsymbol{u}_{\varepsilon} \cdot \boldsymbol{\nabla}\left(J_{\varepsilon} \boldsymbol{\sigma}_{\varepsilon}\right)\right]-\boldsymbol{u}_{\varepsilon} \cdot D^{\alpha} \boldsymbol{\nabla}\left(J_{\varepsilon} \boldsymbol{\sigma}_{\varepsilon}\right)\right\|_{0} .
$$

We then invoke the Sobolev calculus inequality (A.7) to get

$$
I_{1} \leq C\left\|D^{\alpha} \boldsymbol{\sigma}_{\varepsilon}\right\|_{0}\left[\left\|\boldsymbol{\nabla} \boldsymbol{u}_{\varepsilon}\right\|_{L^{\infty}}\left\|\boldsymbol{\nabla}\left(J_{\varepsilon} \boldsymbol{\sigma}_{\varepsilon}\right)\right\|_{m-1}+\left\|\boldsymbol{u}_{\varepsilon}\right\|_{m}\left\|\boldsymbol{\nabla}\left(J_{\varepsilon} \boldsymbol{\sigma}_{\varepsilon}\right)\right\|_{L^{\infty}}\right] .
$$

Using repeatedly the property (A.3) of $J_{\varepsilon}$, the Sobolev embedding Theorem (A.9), and the $\mathrm{CZ}$ inequality, we get the bound

$$
I_{1} \leq \frac{C}{\nu_{s}}\left\|\boldsymbol{\sigma}_{\varepsilon}\right\|_{m}^{3} .
$$

Note that in order to bound $\left\|\boldsymbol{\nabla} \boldsymbol{\sigma}_{\varepsilon}\right\|_{L^{\infty}}$ by $\left\|\boldsymbol{\sigma}_{\varepsilon}\right\|_{m}$ we need $m>5 / 2$.

We turn to $I_{2}$, where we use the Cauchy-Schwarz inequality, the Banach algebra property of $H^{k}\left(\mathbb{R}^{3}\right)$ for $k>3 / 2$, and the CZ inequality,

$$
\begin{aligned}
I_{2} & =2\left(D^{\alpha} \boldsymbol{\sigma}_{\varepsilon}, D^{\alpha}\left[\boldsymbol{\sigma}_{\varepsilon}\left(\boldsymbol{\nabla} \boldsymbol{u}_{\varepsilon}\right)\right]\right) \leq 2\left\|\boldsymbol{\sigma}_{\varepsilon}\right\|_{m}\left\|\boldsymbol{\sigma}_{\varepsilon}\left(\boldsymbol{\nabla} \boldsymbol{u}_{\varepsilon}\right)\right\|_{m} \\
& \leq C\left\|\boldsymbol{\sigma}_{\varepsilon}\right\|_{m}^{2}\left\|\boldsymbol{\nabla} \boldsymbol{u}_{\varepsilon}\right\|_{m} \leq \frac{C}{\nu_{s}}\left\|\boldsymbol{\sigma}_{\varepsilon}\right\|_{m}^{3} .
\end{aligned}
$$

There remains $I_{3}$, which we estimate using the Cauchy-Schwarz inequality followed by the CZ inequality,

$$
I_{3}=\frac{2 \nu_{p}}{\lambda}\left(D^{\alpha} \boldsymbol{\sigma}_{\varepsilon}, D^{\alpha} \boldsymbol{\nabla} \boldsymbol{u}_{\varepsilon}\right) \leq C \frac{\nu_{p}}{\lambda}\left\|D^{\alpha} \boldsymbol{\sigma}_{\varepsilon}\right\|_{0}\left\|D^{\alpha} \boldsymbol{\nabla} \boldsymbol{u}_{\varepsilon}\right\|_{0} \leq C \frac{\nu_{p}}{\lambda \nu_{s}}\left\|\boldsymbol{\sigma}_{\varepsilon}\right\|_{m}^{2} .
$$

Combining the three estimates (3.8), (3.9) and (3.10) we obtain an energy inequality

$$
\frac{d}{d t}\left\|\boldsymbol{\sigma}_{\varepsilon}\right\|_{m}+\frac{1}{\lambda}\left\|\boldsymbol{\sigma}_{\varepsilon}\right\|_{m} \leq c_{1} \frac{\nu_{p}}{\lambda \nu_{s}}\left\|\boldsymbol{\sigma}_{\varepsilon}\right\|_{m}+\frac{c_{2}}{\nu_{s}}\left\|\boldsymbol{\sigma}_{\varepsilon}\right\|_{m}^{2},
$$

from which we conclude the existence of a time $T=T\left(\left\|\sigma_{0}\right\|_{m}\right)$, independent of $\varepsilon$, for which all the $\boldsymbol{\sigma}_{\varepsilon}$ exist and have a common bound in $H^{m}\left(\mathbb{R}^{3}\right)$,

$$
\sup _{0 \leq t \leq T}\left\|\boldsymbol{\sigma}_{\varepsilon}\right\|_{m} \leq \frac{c_{3} e^{c_{3} T}\left\|\boldsymbol{\sigma}_{0}\right\|_{m}}{c_{3}+c_{4}\left(1-e^{c_{3} T}\right)\left\|\boldsymbol{\sigma}_{0}\right\|_{m}} \equiv K\left(\left\|\boldsymbol{\sigma}_{0}\right\|_{m}, T\right),
$$


where $c_{3}=\lambda^{-1}\left(c_{1} \nu_{p} / \nu_{s}-1\right), c_{4}=c_{2} / \nu_{s}$ and $T<\frac{1}{\left|c_{3}\right|} \log \left(1+\left|c_{3}\right| / c_{4}\left\|\boldsymbol{\sigma}_{0}\right\|_{m}\right)$. This concludes the proof of Proposition 3.3.

REMARK 3.6. Substituting back the dimensional parameters, our expression for the uniform existence time is

$$
T<\frac{\lambda}{\left|c_{1} \nu_{p} / \nu_{s}-1\right|} \log \left(1+\frac{\nu_{s}\left|c_{1} \nu_{p} / \nu_{s}-1\right|}{c_{2} \lambda\left\|\boldsymbol{\sigma}_{0}\right\|_{m}}\right) .
$$

First, it follows that this time remains finite in the $\lambda \rightarrow \infty$ limit (i.e., infinite Weissenberg number), in which case $T<\nu_{s} / c_{2}\left\|\sigma_{0}\right\|_{m}$. Second, this time is unbounded (hence, global-in-time existence follows) if $\nu_{s} / \nu_{p}>c_{2}$ and the initial data are suffciently small, namely $\left\|\boldsymbol{\sigma}_{0}\right\|_{m}<\left|c_{3}\right| / c_{4}$.

3.3. Convergence of $\sigma_{\varepsilon}$ in $C\left([0, T], L^{2}\left(\mathbb{R}^{3}\right)\right)$. We proceed to prove Proposition 3.4, whereby the sequence $\boldsymbol{\sigma}_{\varepsilon}$ forms, as $\varepsilon \rightarrow 0$, a Cauchy sequence in the space $C\left([0, T] ; L^{2}\left(\mathbb{R}^{3}\right)\right)$. Here, $T$ is the uniform existence time established in the previous subsection. Specifically, we show that for $\boldsymbol{\sigma}_{\varepsilon}, \boldsymbol{\sigma}_{\varepsilon^{\prime}} \in H^{m}\left(\mathbb{R}^{3}\right)$ solutions of (3.4) with the same initial condition $\boldsymbol{\sigma}_{0}$, the following holds:

$$
\sup _{0 \leq t \leq T}\left\|\boldsymbol{\sigma}_{\varepsilon}-\boldsymbol{\sigma}_{\varepsilon^{\prime}}\right\|_{0} \leq C\left(\left\|\boldsymbol{\sigma}_{0}\right\|_{m}, T\right) \max \left(\varepsilon, \varepsilon^{\prime}\right) .
$$

Hence follows the existence of $\boldsymbol{\sigma} \in C\left([0, T], L^{2}\left(\mathbb{R}^{3}\right)\right)$, such that

$$
\lim _{\varepsilon \rightarrow 0} \sup _{0 \leq t \leq T}\left\|\boldsymbol{\sigma}_{\varepsilon}(\cdot, t)-\boldsymbol{\sigma}(\cdot, t)\right\|_{0}=0 .
$$

As in the previous subsection, we start with an energy equation, this time for the difference $\boldsymbol{\sigma}_{\varepsilon}-\boldsymbol{\sigma}_{\varepsilon^{\prime}}$ :

$$
\begin{aligned}
& \frac{1}{2} \frac{d}{d t}\left\|\boldsymbol{\sigma}_{\varepsilon}-\boldsymbol{\sigma}_{\varepsilon^{\prime}}\right\|_{0}^{2}+\frac{1}{\lambda}\left\|\boldsymbol{\sigma}_{\varepsilon}-\boldsymbol{\sigma}_{\varepsilon^{\prime}}\right\|_{0}^{2} \\
= & -\left(J_{\varepsilon}\left[\boldsymbol{u}_{\varepsilon} \cdot \nabla\left(J_{\varepsilon} \boldsymbol{\sigma}_{\varepsilon}\right)\right]-J_{\varepsilon^{\prime}}\left[\boldsymbol{u}_{\varepsilon^{\prime}} \cdot \boldsymbol{\nabla}\left(J_{\varepsilon^{\prime}} \boldsymbol{\sigma}_{\varepsilon^{\prime}}\right)\right], \boldsymbol{\sigma}_{\varepsilon}-\boldsymbol{\sigma}_{\varepsilon^{\prime}}\right) \\
& +2\left(\boldsymbol{\sigma}_{\varepsilon} \boldsymbol{\nabla} \boldsymbol{u}_{\varepsilon}-\boldsymbol{\sigma}_{\varepsilon^{\prime}} \boldsymbol{\nabla} \boldsymbol{u}_{\varepsilon^{\prime}}, \boldsymbol{\sigma}_{\varepsilon}-\boldsymbol{\sigma}_{\varepsilon^{\prime}}\right) \\
& +\frac{2 \nu_{p}}{\lambda}\left(\boldsymbol{\nabla} \boldsymbol{u}_{\varepsilon}-\boldsymbol{\nabla} \boldsymbol{u}_{\varepsilon^{\prime}}, \boldsymbol{\sigma}_{\varepsilon}-\boldsymbol{\sigma}_{\varepsilon^{\prime}}\right) \equiv I_{1}+I_{2}+I_{3} .
\end{aligned}
$$

$I_{2}$ and $I_{3}$ are easily estimated by the same manipulations as in the previous subsection,

$$
\begin{aligned}
I_{2} & \leq 2\left\|\boldsymbol{\sigma}_{\varepsilon} \boldsymbol{\nabla} \boldsymbol{u}_{\varepsilon}-\boldsymbol{\sigma}_{\varepsilon^{\prime}} \boldsymbol{\nabla} \boldsymbol{u}_{\varepsilon^{\prime}}\right\|_{0}\left\|\boldsymbol{\sigma}_{\varepsilon}-\boldsymbol{\sigma}_{\varepsilon^{\prime}}\right\|_{0} \\
& =2\left\|\left(\boldsymbol{\sigma}_{\varepsilon}-\boldsymbol{\sigma}_{\varepsilon^{\prime}}\right) \boldsymbol{\nabla} \boldsymbol{u}_{\varepsilon}-\boldsymbol{\sigma}_{\varepsilon^{\prime}}\left(\boldsymbol{\nabla} \boldsymbol{u}_{\varepsilon^{\prime}}-\nabla \boldsymbol{u}_{\varepsilon}\right)\right\|_{0}\left\|\boldsymbol{\sigma}_{\varepsilon}-\boldsymbol{\sigma}_{\varepsilon^{\prime}}\right\|_{0} \\
& \leq 2\left[\left\|\boldsymbol{\sigma}_{\varepsilon}-\boldsymbol{\sigma}_{\varepsilon^{\prime}}\right\|\left\|_{0}\right\| \boldsymbol{\nabla} \boldsymbol{u}_{\varepsilon}\left\|_{L^{\infty}}+\right\| \boldsymbol{\sigma}_{\varepsilon^{\prime}}\left\|_{L^{\infty}}\right\| \boldsymbol{\nabla} \boldsymbol{u}_{\varepsilon^{\prime}}-\boldsymbol{\nabla} \boldsymbol{u}_{\varepsilon} \|_{0}\right]\left\|\boldsymbol{\sigma}_{\varepsilon}-\boldsymbol{\sigma}_{\varepsilon^{\prime}}\right\|_{0} \\
& \leq C\left(\left\|\boldsymbol{\nabla} \boldsymbol{u}_{\varepsilon}\right\|_{L^{\infty}}+\left\|\boldsymbol{\sigma}_{\varepsilon^{\prime}}\right\|_{L^{\infty}}\right)\left\|\boldsymbol{\sigma}_{\varepsilon}-\boldsymbol{\sigma}_{\varepsilon^{\prime}}\right\|_{0}^{2} \\
& \leq C\left(\left\|\boldsymbol{\sigma}_{\varepsilon}\right\|_{m}+\left\|\boldsymbol{\sigma}_{\varepsilon^{\prime}}\right\|_{m}\right)\left\|\boldsymbol{\sigma}_{\varepsilon}-\boldsymbol{\sigma}_{\varepsilon^{\prime}}\right\|_{0}^{2}
\end{aligned}
$$

and similarly,

$$
I_{3} \leq \frac{2 \nu_{p}}{\lambda}\left\|\nabla \boldsymbol{u}_{\varepsilon}-\nabla \boldsymbol{u}_{\varepsilon^{\prime}}\right\|_{0}\left\|\boldsymbol{\sigma}_{\varepsilon}-\boldsymbol{\sigma}_{\varepsilon^{\prime}}\right\|_{0} \leq C\left\|\boldsymbol{\sigma}_{\varepsilon}-\boldsymbol{\sigma}_{\varepsilon^{\prime}}\right\|_{0}^{2} .
$$

There remains the advection term $I_{1}$, which we first split as follows:

$$
\begin{aligned}
-I_{1}= & \left(\left(J_{\varepsilon}-J_{\varepsilon^{\prime}}\right)\left[\boldsymbol{u}_{\varepsilon} \cdot \boldsymbol{\nabla}\left(J_{\varepsilon} \boldsymbol{\sigma}_{\varepsilon}\right)\right], \boldsymbol{\sigma}_{\varepsilon}-\boldsymbol{\sigma}_{\varepsilon^{\prime}}\right)+\left(J_{\varepsilon^{\prime}}\left[\left(\boldsymbol{u}_{\varepsilon}-\boldsymbol{u}_{\varepsilon^{\prime}}\right) \cdot \boldsymbol{\nabla}\left(J_{\varepsilon} \boldsymbol{\sigma}_{\varepsilon}\right)\right], \boldsymbol{\sigma}_{\varepsilon}-\boldsymbol{\sigma}_{\varepsilon^{\prime}}\right) \\
& +\left(J_{\varepsilon^{\prime}}\left[\boldsymbol{u}_{\varepsilon^{\prime}} \cdot \boldsymbol{\nabla}\left(J_{\varepsilon}-J_{\varepsilon^{\prime}}\right) \boldsymbol{\sigma}_{\varepsilon}\right], \boldsymbol{\sigma}_{\varepsilon}-\boldsymbol{\sigma}_{\varepsilon^{\prime}}\right)+\left(J_{\varepsilon^{\prime}}\left[\boldsymbol{u}_{\varepsilon} \cdot \boldsymbol{\nabla} J_{\varepsilon^{\prime}}\left(\boldsymbol{\sigma}_{\varepsilon}-\boldsymbol{\sigma}_{\varepsilon^{\prime}}\right)\right], \boldsymbol{\sigma}_{\varepsilon}-\boldsymbol{\sigma}_{\varepsilon^{\prime}}\right) .
\end{aligned}
$$


The last term vanishes because $\boldsymbol{u}_{\varepsilon}$ is divergence-free. For the first three terms we use the Cauchy-Schwarz inequality, obtaining thus

$$
\begin{aligned}
\frac{\left|I_{1}\right|}{\left\|\boldsymbol{\sigma}_{\varepsilon}-\boldsymbol{\sigma}_{\varepsilon^{\prime}}\right\|_{0}} \leq & \left\|\left(J_{\varepsilon}-J_{\varepsilon^{\prime}}\right)\left[\boldsymbol{u}_{\varepsilon} \cdot \nabla\left(J_{\varepsilon} \boldsymbol{\sigma}_{\varepsilon}\right)\right]\right\|_{0}+\left\|J_{\varepsilon^{\prime}}\left[\left(\boldsymbol{u}_{\varepsilon}-\boldsymbol{u}_{\varepsilon^{\prime}}\right) \cdot \nabla J_{\varepsilon} \boldsymbol{\sigma}_{\varepsilon}\right]\right\|_{0} \\
& +\left\|J_{\varepsilon^{\prime}}\left[\boldsymbol{u}_{\varepsilon^{\prime}} \cdot \boldsymbol{\nabla}\left(J_{\varepsilon}-J_{\varepsilon^{\prime}}\right) \boldsymbol{\sigma}_{\varepsilon}\right]\right\|_{0} \equiv A_{1}+A_{2}+A_{3} .
\end{aligned}
$$

By (A.3) the outer $J_{\varepsilon^{\prime}}$ can be replaced in $A_{2}, A_{3}$ by a constant prefactor. $A_{2}$ is easily estimated by

$$
A_{2} \leq C\left\|\boldsymbol{u}_{\varepsilon}-\boldsymbol{u}_{\varepsilon^{\prime}}\right\|_{0}\left\|\boldsymbol{\nabla}\left(J_{\varepsilon} \boldsymbol{\sigma}_{\varepsilon}\right)\right\|_{L^{\infty}} \leq C\left\|\boldsymbol{\sigma}_{\varepsilon}-\boldsymbol{\sigma}_{\varepsilon^{\prime}}\right\|_{0}\left\|\boldsymbol{\sigma}_{\varepsilon}\right\|_{m},
$$

where we have used the $\mathrm{CZ}$ inequality and the Sobolev embedding theorem, with $m>5 / 2$ (see Appendix).

Note that the two remaining terms have the factor $\left(J_{\varepsilon}-J_{\varepsilon^{\prime}}\right)$, which is "small" in the following sense. By (A.2) it follows that

$$
\left\|\left(J_{\varepsilon}-J_{\varepsilon^{\prime}}\right) f\right\|_{0} \leq\left\|\left(J_{\varepsilon}-I\right) f\right\|_{0}+\left\|\left(J_{\varepsilon^{\prime}}-I\right) f\right\|_{0} \leq C\|f\|_{1} \max \left(\varepsilon, \varepsilon^{\prime}\right) .
$$

Thus, $A_{1}$ can be estimated by

$$
A_{1} \leq C\left\|\boldsymbol{u}_{\varepsilon} \cdot \boldsymbol{\nabla}\left(J_{\varepsilon} \boldsymbol{\sigma}_{\varepsilon}\right)\right\|_{1} \max \left(\varepsilon, \varepsilon^{\prime}\right) \leq C\left\|\boldsymbol{\sigma}_{\varepsilon}\right\|_{m}^{2} \max \left(\varepsilon, \varepsilon^{\prime}\right),
$$

where the last inequality follows from the very rough estimate of the $H^{1}$-norm by the $H^{m-1}$ norm, and the Banach algebra property of $H^{k}\left(\mathbb{R}^{3}\right)$ for $k>3 / 2$.

$A_{3}$ verifies an estimate similar to $A_{1}$. Gathering the expressions for $A_{1}, A_{2}, A_{3}$, $I_{2}, I_{3}$, and substituting into the energy equation (3.12),

$$
\frac{1}{2} \frac{d}{d t}\left\|\boldsymbol{\sigma}_{\varepsilon}-\boldsymbol{\sigma}_{\varepsilon^{\prime}}\right\|_{0}^{2} \leq C\left\|\boldsymbol{\sigma}_{\varepsilon}\right\|_{m}^{2} \max \left(\varepsilon, \varepsilon^{\prime}\right)\left\|\boldsymbol{\sigma}_{\varepsilon}-\boldsymbol{\sigma}_{\varepsilon^{\prime}}\right\|_{0}+C\left(\left\|\boldsymbol{\sigma}_{\varepsilon^{\prime}}\right\|_{m}+\left\|\boldsymbol{\sigma}_{\varepsilon}\right\|_{m}\right)\left\|\boldsymbol{\sigma}_{\varepsilon}-\boldsymbol{\sigma}_{\varepsilon^{\prime}}\right\|_{0}^{2} .
$$

We now use the uniform bound (3.11) to obtain

$$
\frac{d}{d t}\left\|\boldsymbol{\sigma}_{\varepsilon}-\boldsymbol{\sigma}_{\varepsilon^{\prime}}\right\|_{0} \leq C\left(\left\|\boldsymbol{\sigma}_{0}\right\|_{m}\right)\left[\max \left(\varepsilon, \varepsilon^{\prime}\right)+\left\|\boldsymbol{\sigma}_{\varepsilon}-\boldsymbol{\sigma}_{\varepsilon^{\prime}}\right\|_{0}\right]
$$

which upon integrating yields

$$
\sup _{0 \leq t \leq T}\left\|\boldsymbol{\sigma}_{\varepsilon}-\boldsymbol{\sigma}_{\varepsilon^{\prime}}\right\|_{0} \leq e^{C\left(\left\|\boldsymbol{\sigma}_{0}\right\|_{m}\right) T} \max \left(\varepsilon, \varepsilon^{\prime}\right),
$$

and we used here the fact that $\boldsymbol{\sigma}_{\varepsilon}$ and $\boldsymbol{\sigma}_{\varepsilon^{\prime}}$ satisfy the same initial conditions. Therefore, $\boldsymbol{\sigma}_{\varepsilon}$ is a Cauchy sequence in the Banach space $C\left([0, T] ; L^{2}\left(\mathbb{R}^{3}\right)\right)$, and hence it has a limit $\boldsymbol{\sigma} \in C\left([0, T] ; L^{2}\left(\mathbb{R}^{3}\right)\right)$. In particular, (3.19) implies that for the limit $\boldsymbol{\sigma}$ we have

$$
\sup _{0 \leq t \leq T}\left\|\boldsymbol{\sigma}_{\varepsilon}-\boldsymbol{\sigma}\right\|_{0} \leq e^{C\left(\left\|\boldsymbol{\sigma}_{0}\right\|_{m}\right) T} \varepsilon
$$

The uniform boundedness (3.11) of the $\boldsymbol{\sigma}_{\varepsilon}$ implies by the Banach-Alaoglu theorem that for every $t \leq T$ the sequence $\boldsymbol{\sigma}_{\varepsilon}(\cdot, t)$ has a subsequence that converges weakly in $H^{m}\left(\mathbb{R}^{3}\right)$. This limit must however coincide with the $L^{2}$ limit, $\boldsymbol{\sigma}(\cdot, t)$. Moreover, the Banach-Alaoglu theorem also implies that

$$
\|\boldsymbol{\sigma}(\cdot, t)\|_{m} \leq \liminf _{\varepsilon \rightarrow 0}\left\|\boldsymbol{\sigma}_{\varepsilon}(\cdot, t)\right\|_{m},
$$

thus it follows from (3.11) that for every $t \in[0, T]$,

$$
\sup _{0 \leq t \leq T}\|\boldsymbol{\sigma}(\cdot, t)\|_{m} \leq K\left(\left\|\boldsymbol{\sigma}_{0}\right\|_{m}, T\right) .
$$

Note, however, that we do not yet know that $\boldsymbol{\sigma}$ is a continuous function from $[0, T]$ into $H^{m}\left(\mathbb{R}^{3}\right)$. It is the task of the next subsection to show that $\boldsymbol{\sigma}$ is in $C\left([0, T], H^{m}\left(\mathbb{R}^{3}\right)\right)$. 
3.4. Continuity in $H^{m}\left(\mathbb{R}^{3}\right)$. In this section we prove Proposition 3.5 , whereby $\boldsymbol{\sigma} \in C\left([0, T], H^{m}\left(\mathbb{R}^{3}\right)\right)$. We do it in several steps.

We start by showing that the mollified solutions $\boldsymbol{\sigma}_{\varepsilon}$ converge to $\boldsymbol{\sigma}$ in all "intermediate" norms, $C\left([0, T], H^{m^{\prime}}\left(\mathbb{R}^{3}\right)\right)$, for all $0<m^{\prime}<m$. For that we invoke the following interpolation lemma in Sobolev space:

$$
\|\boldsymbol{\tau}\|_{m^{\prime}} \leq C_{m}\|\boldsymbol{\tau}\|_{0}^{1-m^{\prime} / m}\|\boldsymbol{\tau}\|_{m}^{m^{\prime} / m}
$$

valid for all $\boldsymbol{\tau} \in H^{m}\left(\mathbb{R}^{3}\right)$ and $0 \leq m^{\prime} \leq m$. Substituting $\boldsymbol{\tau}=\boldsymbol{\sigma}_{\varepsilon}-\boldsymbol{\sigma}$,

$$
\left\|\boldsymbol{\sigma}_{\varepsilon}-\boldsymbol{\sigma}\right\|_{m^{\prime}} \leq C_{m}\left\|\boldsymbol{\sigma}_{\varepsilon}-\boldsymbol{\sigma}\right\|_{0}^{1-m^{\prime} / m}\left\|\boldsymbol{\sigma}_{\varepsilon}-\boldsymbol{\sigma}\right\|_{m}^{m^{\prime} / m} .
$$

Using the uniform boundedness (3.11) of $\boldsymbol{\sigma}_{\varepsilon}$ and (3.20),

$$
\left\|\boldsymbol{\sigma}_{\varepsilon}-\boldsymbol{\sigma}\right\|_{m}^{m^{\prime} / m} \leq\left(\left\|\boldsymbol{\sigma}_{\varepsilon}\right\|_{m}+\|\boldsymbol{\sigma}\|_{m}\right)^{m^{\prime} / m} \leq\left[2 K\left(\left\|\boldsymbol{\sigma}_{0}\right\|_{m}, T\right)\right]^{m^{\prime} / m} ;
$$

thus we obtain

$$
\sup _{0 \leq t \leq T}\left\|\boldsymbol{\sigma}_{\varepsilon}(\cdot, t)-\boldsymbol{\sigma}(\cdot, t)\right\|_{m^{\prime}} \leq\left[2 K\left(\left\|\boldsymbol{\sigma}_{0}\right\|_{m}, T\right)\right]^{m^{\prime} / m} \sup _{0 \leq t \leq T}\left\|\boldsymbol{\sigma}_{\varepsilon}(\cdot, t)-\boldsymbol{\sigma}(\cdot, t)\right\|_{0}^{1-m^{\prime} / m} ;
$$

i.e., uniform convergence $\boldsymbol{\sigma}_{\varepsilon} \rightarrow \boldsymbol{\sigma}$ in all intermediate norms.

To show that $\boldsymbol{\sigma}$ is time-continuous in the highest norm, we first show that $\boldsymbol{\sigma}$ is time-continuous in the weak topology of $H^{m}\left(\mathbb{R}^{3}\right)$. That is, we claim that for every $\phi \in H^{-m}\left(\mathbb{R}^{3}\right)$,

$$
g(t)=\langle\phi, \boldsymbol{\sigma}(\cdot, t)\rangle
$$

is continuous in time, where $\langle\cdot, \cdot\rangle$ is the dual pairing between $H^{m}\left(\mathbb{R}^{3}\right)$ and $H^{-m}\left(\mathbb{R}^{3}\right)$. Since $\sigma$ is time-continuous in the strong topologies of all the intermediate norms, it follows that $\langle\phi, \boldsymbol{\sigma}(\cdot, t)\rangle$ is time-continuous for all $\phi \in H^{-m^{\prime}}\left(\mathbb{R}^{3}\right)$, but since the latter is dense in $H^{-m}\left(\mathbb{R}^{3}\right)$ and $\boldsymbol{\sigma}$ satisfies the uniform bound (3.20) in $H^{m}$, the continuity of $g(t)$ follows.

As is well known, continuity in the weak topology of a Hilbert space supplemented by the continuity of the norm yields continuity in the strong topology. Thus, it remains to show that $\|\boldsymbol{\sigma}(\cdot, t)\|_{m}$ is time-continuous.

We start by showing continuity at the initial time $t=0$. For $h>0$ we have

$$
\left\|\boldsymbol{\sigma}(\cdot, h)-\boldsymbol{\sigma}_{0}\right\|_{m}^{2}=\|\boldsymbol{\sigma}(\cdot, h)\|_{m}^{2}-\left\|\boldsymbol{\sigma}_{0}\right\|_{m}^{2}-2\left(\boldsymbol{\sigma}(\cdot, h)-\boldsymbol{\sigma}_{0}, \boldsymbol{\sigma}_{0}\right)_{m},
$$

where $(\cdot, \cdot)_{m}$ denotes the inner product in $H^{m}\left(\mathbb{R}^{3}\right)$. As $h \rightarrow 0^{+}$, the last term vanishes by the time-continuity of $\boldsymbol{\sigma}$ in the weak topology in $H^{m}\left(\mathbb{R}^{3}\right)$. This yields

$$
\left\|\boldsymbol{\sigma}_{0}\right\|_{m} \leq \liminf _{h \searrow 0}\|\boldsymbol{\sigma}(\cdot, h)\|_{m} .
$$

To obtain the reverse inequality we observe that by following similar steps as in the above proof one can obtain the following modification of (3.20),

$$
\sup _{0 \leq t \leq \tau}\|\boldsymbol{\sigma}(\cdot, t)\|_{m} \leq K\left(\left\|\boldsymbol{\sigma}_{0}\right\|_{m}, \tau\right),
$$

for all $\tau \in(0, T]$, where $K$ is given by (3.20). Therefore, by taking $\tau=h$ and assuming $h$ is small enough, the above inequality and (3.20) yields

$$
\|\boldsymbol{\sigma}(\cdot, h)\|_{m} \leq\left\|\boldsymbol{\sigma}_{0}\right\|_{m}+C h\left\|\boldsymbol{\sigma}_{0}\right\|_{m}\left(1+\left\|\boldsymbol{\sigma}_{0}\right\|_{m}\right) .
$$


Since $\|\boldsymbol{\sigma}(\cdot, h)\|_{m}$ is bounded we may let $h \rightarrow 0^{+}$, obtaining

$$
\underset{h \searrow 0}{\limsup }\|\boldsymbol{\sigma}(\cdot, h)\|_{m} \leq\left\|\boldsymbol{\sigma}_{0}\right\|_{m}
$$

which together with (3.21) implies right-continuity at $t=0$; left-continuity at $t=0$ follows from the fact that the Oldroyd-B system can be time reversed (unlike parabolic equations such as the viscous Navier-Stokes equations).

It remains to show that $\boldsymbol{\sigma}$ is time continuous at any arbitrary time $s \in[0, T]$. We use the fact that $\boldsymbol{\sigma}(\cdot, s) \in H^{m}\left(\mathbb{R}^{3}\right)$ to construct a new set of mollified solutions with initial data at $t=s, \tilde{\boldsymbol{\sigma}}_{\varepsilon}(\cdot, s)=\boldsymbol{\sigma}(\cdot, s)$. By the same line of reasoning as before, these solutions converge in $C\left([0, T], L^{2}\left(\mathbb{R}^{3}\right)\right)$ to a solution $\tilde{\boldsymbol{\sigma}}$ which belongs, at all times, to $H^{m}\left(\mathbb{R}^{3}\right)$ and is continuous at the initial time $t=s$. Let $\boldsymbol{\sigma}_{\varepsilon}(\cdot, t)$ be as before. Now, one can follow the same steps as in Sec. 3.3 to show that

$$
\begin{array}{r}
\frac{1}{2} \frac{d}{d t}\left\|\boldsymbol{\sigma}_{\varepsilon}-\tilde{\boldsymbol{\sigma}}_{\varepsilon}\right\|_{0}^{2} \leq C\left(\left\|\tilde{\boldsymbol{\sigma}}_{\varepsilon}\right\|_{m}+\left\|\boldsymbol{\sigma}_{\varepsilon}\right\|_{m}\right)\left\|\boldsymbol{\sigma}_{\varepsilon}\right\|_{m} \varepsilon\left\|\boldsymbol{\sigma}_{\varepsilon}-\tilde{\boldsymbol{\sigma}}_{\varepsilon}\right\|_{0} \\
+C\left(\left\|\tilde{\boldsymbol{\sigma}}_{\varepsilon}\right\|_{m}+\left\|\boldsymbol{\sigma}_{\varepsilon}\right\|_{m}\right)\left\|\boldsymbol{\sigma}_{\varepsilon}-\tilde{\boldsymbol{\sigma}}_{\varepsilon}\right\|_{0}^{2}
\end{array}
$$

(cf. (3.18)). Similar steps show that around $t=s$

$$
\left\|\tilde{\boldsymbol{\sigma}}_{\varepsilon}\right\|_{m} \leq K\left(\left\|\boldsymbol{\sigma}_{\varepsilon}(\cdot, s)\right\|_{m}\right) .
$$

Therefore, we have

$$
\frac{d}{d t}\left\|\boldsymbol{\sigma}_{\varepsilon}-\tilde{\boldsymbol{\sigma}}_{\varepsilon}\right\|_{0} \leq C\left(\left\|\boldsymbol{\sigma}_{\varepsilon}(\cdot, s)\right\|_{m}\right)\left(\varepsilon+\left\|\boldsymbol{\sigma}_{\varepsilon}-\tilde{\boldsymbol{\sigma}}_{\varepsilon}\right\|_{0}\right) .
$$

Integrating, we obtain

$$
\left\|\boldsymbol{\sigma}_{\varepsilon}(\cdot, t)-\tilde{\boldsymbol{\sigma}}_{\varepsilon}(\cdot, t)\right\|_{0} \leq e^{C\left(\left\|\boldsymbol{\sigma}_{\varepsilon}(\cdot, s)\right\|_{m}\right)(t-s)}\left\|\boldsymbol{\sigma}_{\varepsilon}(\cdot, s)-\tilde{\boldsymbol{\sigma}}_{\varepsilon}(\cdot, s)\right\|_{0}+e^{C\left(\left\|\boldsymbol{\sigma}_{\varepsilon}(\cdot, s)\right\|_{m}\right)(t-s)} \varepsilon .
$$

Now, as we let $\varepsilon \rightarrow 0$, we know that $\boldsymbol{\sigma}_{\varepsilon}(\cdot, s) \rightarrow \boldsymbol{\sigma}(\cdot, s)$, and we know that $\tilde{\boldsymbol{\sigma}}_{\varepsilon}(\cdot, s)=$ $\boldsymbol{\sigma}(\cdot, s)$; thus we conclude that

$$
\|\boldsymbol{\sigma}(\cdot, t)-\tilde{\boldsymbol{\sigma}}(\cdot, t)\|_{0}=0
$$

Since $\tilde{\boldsymbol{\sigma}}$ is continuous at $t=s$, so is $\boldsymbol{\sigma}$.

3.5. $\boldsymbol{\sigma}$ is a solution of (2.4). Having shown that $\boldsymbol{\sigma} \in C\left([0, T], H^{m}\left(\mathbb{R}^{3}\right)\right)$, it remains to show that $\boldsymbol{\sigma}$ is indeed a solution of (2.4) and that

$$
\boldsymbol{\sigma} \in C\left([0, T], H^{m}\left(\mathbb{R}^{3}\right)\right) \cap C^{1}\left([0, T], H^{m-1}\left(\mathbb{R}^{3}\right)\right) .
$$

To show that, we refer once more to the mollified solutions, whose evolution satifies the integral equation,

$$
\boldsymbol{\sigma}_{\varepsilon}(\cdot, t)=\boldsymbol{\sigma}_{0}+\int_{0}^{t} \boldsymbol{F}_{\varepsilon}\left(\boldsymbol{\sigma}_{\varepsilon}(\cdot, s)\right) d s .
$$

We now exploit the convergence of $\boldsymbol{\sigma}_{\varepsilon}$ to $\boldsymbol{\sigma}$ in all the intermediate norms. Specifically, we set $5 / 2<m^{\prime}<m$, and claim that

$$
\boldsymbol{\sigma}_{\varepsilon} \rightarrow \boldsymbol{\sigma} \quad \text { in } C\left([0, T], H^{m^{\prime}}\left(\mathbb{R}^{3}\right)\right)
$$


implies that

$$
\begin{aligned}
& J_{\varepsilon}\left[\boldsymbol{u}_{\varepsilon} \cdot \boldsymbol{\nabla}\left(J_{\varepsilon} \boldsymbol{\sigma}_{\varepsilon}\right)\right] \rightarrow \boldsymbol{u} \cdot \boldsymbol{\nabla} \boldsymbol{\sigma}, \\
& \boldsymbol{\sigma}_{\varepsilon}\left(\boldsymbol{\nabla} \boldsymbol{u}_{\varepsilon}\right) \rightarrow \boldsymbol{\sigma}(\boldsymbol{\nabla} \boldsymbol{u}), \\
& \boldsymbol{\nabla} \boldsymbol{u}_{\varepsilon} \rightarrow \boldsymbol{\nabla} \boldsymbol{u}
\end{aligned}
$$

in $C\left([0, T], H^{m^{\prime}-1}\left(\mathbb{R}^{3}\right)\right)$. The last two identities follow from the CZ inequality (A.11) and the Banach algebra property of $H^{k}\left(\mathbb{R}^{3}\right)$ for $k>3 / 2$. The convergence of the advection term follows from the same considerations, up to the loss of one order of regularity due to the gradient of $\boldsymbol{\sigma}_{\varepsilon}$. Thus,

$$
\boldsymbol{\sigma}(\cdot, t)=\boldsymbol{\sigma}_{0}+\int_{0}^{t} \boldsymbol{F}(\boldsymbol{\sigma}(\cdot, s)) d s,
$$

which proves that $\boldsymbol{\sigma} \in C^{1}\left([0, T], H^{m-1}\left(\mathbb{R}^{3}\right)\right)$, and satisfies the differential equation (2.4).

REMARK 3.7. Based on the previous remark, this also proves global-in-time existence for small initial data.

\section{A Beale-Kato-Majda breakdown condition}

Having proved the local-in-time existence of solutions to the Oldroyd-B Equation (2.1), or its ODE representation (2.4), we turn to the main purpose of this paper, which is the characterization of the breakdown of such solutions at finite time. By the continuation theorem for autonomous ODEs, if $T^{*}<\infty$ and $\left[0, T^{*}\right)$ is the maximal time of existence of the solution $\sigma$, then

$$
\limsup _{t \nearrow T^{*}}\|\boldsymbol{\sigma}\|_{m}=\infty
$$

Such a breakdown criterion is not informative enough, as it roughly says that "a solution exists as long as it exists". Our main theorem below provides a more concise breakdown condition, which is only associated with the stress itself, and does not involve any of its derivatives:

Theorem 4.1. Let $\boldsymbol{\sigma}$ be a local-in-time solution to (2.1) in the class

$$
C\left([0, T) ; H^{m}\left(\mathbb{R}^{3}\right)\right) \cap C^{1}\left([0, T) ; H^{m-1}\left(\mathbb{R}^{3}\right)\right),
$$

with $m \geq 3$. Suppose that $\left[0, T^{*}\right)$ is the maximal time of existence, with $T^{*}<\infty$; then

$$
\lim _{t \nearrow T^{*}} \int_{0}^{t}\|\boldsymbol{\sigma}(\cdot, s)\|_{L^{\infty}} d s=\infty
$$

The proof is similar in essence to the proof of the Beale-Kato-Majda theorem for the Euler equations [6]. All that is needed is an a priori estimate of the form,

$$
\|\boldsymbol{\sigma}(\cdot, t)\|_{m} \leq C\left(t, \int_{0}^{t}\|\boldsymbol{\sigma}(\cdot, s)\|_{L^{\infty}} d s\right)
$$

where $C$ is a continuous function of its arguments, hence (4.1) occurs only if (4.2) occurs. The estimate (4.3) is derived in two steps, detailed in the next two subsections. 
4.1. A priori estimates for the $H^{m}\left(\mathbb{R}^{3}\right)$ norm. Our estimates rely on the following version of the Gagliardo-Nirenberg inequality,

$$
\left\|D^{k} f\right\|_{L^{q}} \leq C\|f\|_{L^{\infty}}^{1-k / m}\left\|D^{m} f\right\|_{L^{k q / m}}^{k / m},
$$

where $1 \leq k \leq m$ and $1<p<\infty$, with $p=\frac{k q}{m}$. With this, we prove the following lemma: Lemma 4.2. For $f, g, h \in H^{m}\left(\mathbb{R}^{3}\right)$ the following triple-product inequality holds,

$$
\int_{\mathbb{R}^{3}}\left|D^{\alpha} h\right|\left|D^{\beta} f\right|\left|D^{\alpha-\beta} g\right| d \boldsymbol{x} \leq C\|h\|_{|\alpha|}\|f\|_{|\alpha|}^{|\beta| /|\alpha|}\|g\|_{|\alpha|}^{|\alpha-\beta| /|\alpha|}\|f\|_{L^{\infty}}^{1-|\beta| /|\alpha|}\|g\|_{L^{\infty}}^{1-|\alpha-\beta| /|\alpha|}
$$

where $\beta<\alpha$.

Proof. We start with the triple product inequality

$$
\int_{\mathbb{R}^{3}}\left|D^{\alpha} h\left\|D^{\beta} f\right\| D^{\alpha-\beta} g\right| d \boldsymbol{x} \leq\left\|D^{\alpha} h\right\|_{0}\left\|D^{\beta} f\right\|_{L^{q}}\left\|D^{\alpha-\beta} g\right\|_{L^{p}},
$$

where $1 / p+1 / q=1 / 2$. We then use twice the Gagliardo-Nirenberg inequality,

$$
\left\|D^{\beta} f\right\|_{L^{q}} \leq C\|f\|_{L^{\infty}}^{1-|\beta| /|\alpha|}\left\|D^{\alpha} f\right\|_{L^{|\beta| q /|\alpha|}}^{|\beta| /|\alpha|}
$$

and

$$
\left\|D^{\alpha-\beta} g\right\|_{L^{p}} \leq C\|g\|_{L^{\infty}}^{1-|\alpha-\beta| /|\alpha|}\left\|D^{\alpha} g\right\|_{L^{|\alpha-\beta| p /|\alpha|}}^{|\alpha-\beta| / \alpha \mid} .
$$

If we choose

$$
q=\frac{2|\alpha|}{|\beta|} \quad \text { and } \quad p=\frac{2|\alpha|}{|\alpha-\beta|}
$$

then (4.4) is obtained.

We are going to make an extensive use of inequality (4.4) for the case where $f=\boldsymbol{\sigma}, g=\boldsymbol{\nabla} \boldsymbol{u}$ and $h=\boldsymbol{\sigma}$ (more precisely, $f, g, h$ are components of these tensors). Then, combined with the CZ inequality (A.11), we get

$$
\left(D^{\alpha} \boldsymbol{\sigma},\left(D^{\beta} \boldsymbol{\sigma}\right)\left[D^{\alpha-\beta}(\boldsymbol{\nabla} \boldsymbol{u})\right]\right) \leq C\|\boldsymbol{\sigma}\|_{|\alpha|}^{2}\|\boldsymbol{\sigma}\|_{L^{\infty}}^{1-|\beta| /|\alpha|}\|\boldsymbol{\nabla} \boldsymbol{u}\|_{L^{\infty}}^{1-|\alpha-\beta| /|\alpha|},
$$

which combined with Young's inequality finally gives:

$$
\left(D^{\alpha} \boldsymbol{\sigma},\left(D^{\beta} \boldsymbol{\sigma}\right)\left[D^{\alpha-\beta}(\boldsymbol{\nabla} \boldsymbol{u})\right]\right) \leq C\|\boldsymbol{\sigma}\|_{|\alpha|}^{2}\left(\|\boldsymbol{\sigma}\|_{L^{\infty}}+\|\boldsymbol{\nabla} \boldsymbol{u}\|_{L^{\infty}}\right) .
$$

For every $|\alpha| \leq m$ the $L^{2}$-norm of the $\alpha$-th derivative of $\boldsymbol{\sigma}$ satisfies the energy equation

$$
\begin{aligned}
\frac{1}{2} \frac{d}{d t}\left\|D^{\alpha} \boldsymbol{\sigma}\right\|_{0}^{2}+\frac{1}{\lambda}\left\|D^{\alpha} \boldsymbol{\sigma}\right\|_{0}^{2}= & -\left(D^{\alpha} \boldsymbol{\sigma}, D^{\alpha}\left(u_{k} \cdot \partial_{k} \boldsymbol{\sigma}\right)\right) \\
& +2\left(D^{\alpha} \boldsymbol{\sigma}, D^{\alpha}[(\boldsymbol{\sigma}(\boldsymbol{\nabla} \boldsymbol{u})])+\frac{2 \nu_{p}}{\lambda}\left(D^{\alpha} \boldsymbol{\sigma}, D^{\alpha}(\boldsymbol{\nabla} \boldsymbol{u})\right) .\right.
\end{aligned}
$$

The last term is easily estimated using the Cauchy-Schwarz inequality and the CZ inequality (A.11),

$$
\left(D^{\alpha} \boldsymbol{\sigma}, D^{\alpha}(\boldsymbol{\nabla} \boldsymbol{u})\right) \leq C\left\|D^{\alpha} \boldsymbol{\sigma}\right\|_{0}^{2} .
$$


The middle term can be written as

$$
\left(D^{\alpha} \boldsymbol{\sigma}, D^{\alpha}[(\boldsymbol{\sigma}(\boldsymbol{\nabla} \boldsymbol{u})])=\sum_{\beta \leq \alpha}\left(D^{\alpha} \boldsymbol{\sigma},\left(D^{\beta} \boldsymbol{\sigma}\right)\left(D^{\alpha-\beta} \boldsymbol{\nabla} \boldsymbol{u}\right)\right),\right.
$$

which is a finite sum of terms, each of which can be bounded using (4.5).

There remains the advection term. Because $\boldsymbol{u}$ is incompressible, the term $u_{k} \partial_{k}\left(D^{\alpha} \boldsymbol{\sigma}\right)$ vanishes, which means that $\boldsymbol{u}$ is differentiated at least once, and we can use (4.5) once again. Thus, we obtain the inequality,

$$
\frac{1}{2} \frac{d}{d t}\left\|D^{\alpha} \boldsymbol{\sigma}\right\|_{0}^{2}+\frac{1}{\lambda}\left\|D^{\alpha} \boldsymbol{\sigma}\right\|_{0}^{2} \leq C\left[1+\|\boldsymbol{\sigma}\|_{L^{\infty}}+\|\boldsymbol{\nabla} \boldsymbol{u}\|_{L^{\infty}}\right]\left\|D^{\alpha} \boldsymbol{\sigma}\right\|_{0}^{2},
$$

and summing up over all $|\alpha| \leq m$,

$$
\frac{d}{d t}\|\boldsymbol{\sigma}\|_{m}+\frac{1}{\lambda}\|\boldsymbol{\sigma}\|_{m} \leq C\left(1+\|\boldsymbol{\sigma}\|_{L^{\infty}}+\|\boldsymbol{\nabla} \boldsymbol{u}\|_{L^{\infty}}\right)\|\boldsymbol{\sigma}\|_{m} .
$$

A simple integration yields:

$$
\|\boldsymbol{\sigma}\|_{m} \leq \exp \left[C \int_{0}^{t}\left(1+\|\boldsymbol{\sigma}\|_{L^{\infty}}+\|\boldsymbol{\nabla} \boldsymbol{u}\|_{L^{\infty}}\right) d s\right]\left\|\boldsymbol{\sigma}_{0}\right\|_{m} .
$$

A comment: the energy inequality (4.6) is only formal since we have not shown that the $H^{m}\left(\mathbb{R}^{3}\right)$ norm of $\boldsymbol{\sigma}$ was differentiable. To rectify this delicacy, one has to carry all estimates with the mollified solutions $\boldsymbol{\sigma}_{\varepsilon}$, which are differentiable in all Sobolev spaces, and take the limit $\varepsilon \rightarrow 0$, only once we have obtained a final estimate for $\left\|\boldsymbol{\sigma}_{\varepsilon}\right\|_{m}$ in the integrated form.

We will need one more estimate. For all indices $i, j, k$ we have

$$
\frac{\partial}{\partial t} \partial_{k} \sigma_{i j}+\frac{1}{\lambda} \partial_{k} \sigma_{i j}=-\partial_{k}\left(u_{l} \partial_{l} \sigma_{i j}\right)+\partial_{k}\left(\sigma_{i l}\left(\partial_{l} u_{j}\right)+\left(\partial_{l} u_{i}\right) \sigma_{l j}\right)+\frac{\nu_{p}}{\lambda} \partial_{k}\left(\partial_{i} u_{j}+\partial_{j} u_{i}\right) .
$$

Multiplying by $\left(\partial_{k} \sigma_{i j}\right)^{3}$ (with summation over all indexes) and integrating over $\mathbb{R}^{3}$ we get

$$
\begin{aligned}
& \frac{1}{4} \frac{d}{d t}\|\boldsymbol{\nabla} \boldsymbol{\sigma}\|_{L^{4}}^{4}+\frac{1}{\lambda}\|\boldsymbol{\nabla} \boldsymbol{\sigma}\|_{L^{4}}^{4} \\
= & -\int_{\mathbb{R}^{3}}\left(\partial_{k} \sigma_{i j}\right)^{3} \partial_{k}\left(u_{l} \partial_{l} \sigma_{i j}\right) d \boldsymbol{x} \\
& +2 \int_{\mathbb{R}^{3}}\left(\partial_{k} \sigma_{i j}\right)^{3} \partial_{k}\left[\sigma_{i l}\left(\partial_{l} u_{j}\right)\right] d \boldsymbol{x}+\frac{2 \nu_{p}}{\lambda} \int_{\mathbb{R}^{3}}\left(\partial_{k} \sigma_{i j}\right)^{3} \partial_{k}\left(\partial_{i} u_{j}\right) d \boldsymbol{x} .
\end{aligned}
$$

Using for the first two terms the triple product inequality, and the Hölder inequality for the third, we get

$$
\begin{aligned}
\frac{1}{4} \frac{d}{d t}\|\boldsymbol{\nabla} \boldsymbol{\sigma}\|_{L^{4}}^{4}+\frac{1}{\lambda}\|\boldsymbol{\nabla} \boldsymbol{\sigma}\|_{L^{4}}^{4} \leq & C\left[\|\boldsymbol{\nabla} \boldsymbol{\sigma}\|_{L^{4}}^{4}\|\boldsymbol{\nabla} \boldsymbol{u}\|_{L^{\infty}}+\|\boldsymbol{\sigma}\|_{L^{\infty}}\|\boldsymbol{\nabla} \boldsymbol{\nabla} \boldsymbol{u}\|_{L^{4}}\|\boldsymbol{\nabla} \boldsymbol{\sigma}\|_{L^{4}}^{3}\right. \\
& \left.+\|\boldsymbol{\nabla} \boldsymbol{\sigma}\|_{L^{4}}^{3}\|\boldsymbol{\nabla} \boldsymbol{\nabla} \boldsymbol{u}\|_{L^{4}}\right]
\end{aligned}
$$

and after applying once again the CZ inequality (A.11),

$$
\frac{d}{d t}\|\boldsymbol{\nabla} \boldsymbol{\sigma}\|_{L^{4}} \leq C\left[1+\|\boldsymbol{\nabla} \boldsymbol{u}\|_{L^{\infty}}+\|\boldsymbol{\sigma}\|_{L^{\infty}}\right]\|\boldsymbol{\nabla} \boldsymbol{\sigma}\|_{L^{4}},
$$

from which we get

$$
\|\boldsymbol{\nabla} \boldsymbol{\sigma}\|_{L^{4}} \leq \exp \left[C \int_{0}^{t}\left(1+\|\boldsymbol{\sigma}\|_{L^{\infty}}+\|\boldsymbol{\nabla} \boldsymbol{u}\|_{L^{\infty}}\right) d s\right]\left\|\nabla \boldsymbol{\sigma}_{0}\right\|_{L^{4}}
$$


4.2. $L^{\infty}$ estimate of $\nabla \boldsymbol{u} . \quad$ So far we have shown in (4.7) that if the solution $\boldsymbol{\sigma}$ breaks down at time $T^{*}$ then,

$$
\lim _{t \nearrow T^{*}} \int_{0}^{t}\left(1+\|\boldsymbol{\sigma}\|_{L^{\infty}}+\|\boldsymbol{\nabla} \boldsymbol{u}\|_{L^{\infty}}\right) d s=\infty .
$$

To complete the proof of Theorem 4.1 it is sufficient to show that

$$
\int_{0}^{t}\left(1+\|\boldsymbol{\sigma}\|_{L^{\infty}}+\|\boldsymbol{\nabla} \boldsymbol{u}\|_{L^{\infty}}\right) d s \leq C\left(t, \int_{0}^{t}\|\boldsymbol{\sigma}\|_{L^{\infty}} d s\right) .
$$

Thus, we need to estimate $\|\boldsymbol{\nabla} \boldsymbol{u}\|_{L^{\infty}}$ in terms of $\|\boldsymbol{\sigma}\|_{L^{\infty}}$. Note that the CZ inequality provides a bound for $\|\boldsymbol{\nabla} \boldsymbol{u}\|_{L^{p}}$ in terms of $\|\boldsymbol{\sigma}\|_{L^{p}}$ for all $p$ (such a bound exists within any of the Sobolev $W^{m, p}$ norms), but the prefactor is linear in $p$, hence $p$ cannot be taken to be infinite. Instead, one has to perform a more delicate analysis.

Consider the integral relation (2.6) between $\boldsymbol{\sigma}$ and $\boldsymbol{\nabla} \boldsymbol{u}$. We split the domain of integration into an "outer domain" $|\boldsymbol{y}|>R$, a "middle annulus" $\varepsilon<|\boldsymbol{y}|<R$, and an "inner disc", $|\boldsymbol{y}|<\varepsilon$, namely,

$$
\boldsymbol{\nabla} \boldsymbol{u}=-\frac{1}{5 \nu_{s}}\left(\boldsymbol{\sigma}-\frac{\boldsymbol{I}}{3} \operatorname{tr} \boldsymbol{\sigma}\right)+\frac{1}{8 \pi \nu_{s}}\left(I_{1}+I_{2}+I_{3}\right),
$$

where

$$
\begin{aligned}
& I_{1}(\boldsymbol{x})=\int_{R<|\boldsymbol{y}|} \boldsymbol{M}^{(2)}(\boldsymbol{y}): \boldsymbol{\sigma}(\boldsymbol{x}-\boldsymbol{y}) d \boldsymbol{y}, \\
& I_{2}(\boldsymbol{x})=\int_{\varepsilon<|\boldsymbol{y}|<R} \boldsymbol{M}^{(2)}(\boldsymbol{y}): \boldsymbol{\sigma}(\boldsymbol{x}-\boldsymbol{y}) d \boldsymbol{y}, \\
& I_{3}(\boldsymbol{x})=(\text { P.V. }) \int_{|\boldsymbol{y}|<\varepsilon} \boldsymbol{M}^{(2)}(\boldsymbol{y}): \boldsymbol{\sigma}(\boldsymbol{x}-\boldsymbol{y}) d \boldsymbol{y} .
\end{aligned}
$$

Recall that $\boldsymbol{M}^{(2)}$ is homogeneous of degree -3 and averages to zero on the unit sphere.

The "outer" integral is estimated using the Cauchy-Schwarz inequality,

$$
\left|I_{1}(\boldsymbol{x})\right| \leq \frac{C}{R^{3 / 2}}\|\boldsymbol{\sigma}\|_{0} .
$$

The "middle" integral is estimated by taking out the infinity norm of the stress:

$$
\left|I_{2}(\boldsymbol{x})\right| \leq C\|\boldsymbol{\sigma}\|_{L^{\infty}} \log \frac{R}{\varepsilon} .
$$

For the "inner" integral, we exploit the fact that $\boldsymbol{M}^{(2)}$ averages to zero on the unit sphere to subtract

$$
(\mathrm{P} . \mathrm{V} .) \int_{|\boldsymbol{y}|<\varepsilon} \boldsymbol{M}^{(2)}(\boldsymbol{y}): \boldsymbol{\sigma}(\boldsymbol{x}) d \boldsymbol{y}=0
$$

so that

$$
I_{3}=(\mathrm{P} . \mathrm{V} .) \int_{|\boldsymbol{y}|<\varepsilon} \boldsymbol{M}^{(2)}(\boldsymbol{y}):[\boldsymbol{\sigma}(\boldsymbol{x}-\boldsymbol{y})-\boldsymbol{\sigma}(\boldsymbol{x})] d \boldsymbol{y} .
$$


By the mean-value theorem (this assumes that $\sigma \in C^{1}\left(\mathbb{R}^{3}\right)$, which is the case since $m>5 / 2$ ), we obtain

$$
\left|I_{3}(\boldsymbol{x}) \leq \int_{|\boldsymbol{y}|<\varepsilon}\right| \boldsymbol{M}^{(2)}(\boldsymbol{y}):[(\boldsymbol{y} \cdot \nabla) \boldsymbol{\sigma}(\boldsymbol{\xi})] \mid d \boldsymbol{y} \leq C\left(\int_{|\boldsymbol{y}|<\varepsilon}\left|M^{(2)}(\boldsymbol{y}) \boldsymbol{y}\right|^{p}\right)^{1 / p}\|\boldsymbol{\nabla} \boldsymbol{\sigma}\|_{L^{q}},
$$

where we used Hölder's inequality, and $\boldsymbol{\xi}$ represents an intermediate point. Since $\boldsymbol{M}^{(2)}$ is homogeneous of degree -3 , this $L^{p}$-norm is finite provided that $2-2 p>-1$, i.e., $p<3 / 2$, and consequently $q>3$. Setting $q=4$ and combining all three contributions, we get

$$
\left|(\mathrm{P} . \mathrm{V} .) \int_{\mathbb{R}^{3}} \boldsymbol{M}^{(2)}(\boldsymbol{y}): \boldsymbol{\sigma}(\boldsymbol{x}-\boldsymbol{y}) d \boldsymbol{y}\right| \leq C\left(\frac{1}{R^{3 / 2}}\|\boldsymbol{\sigma}\|_{0}+\|\boldsymbol{\nabla} \boldsymbol{\sigma}\|_{L^{4}} \varepsilon^{1 / 4}+\|\boldsymbol{\sigma}\|_{L^{\infty}} \log \frac{R}{\varepsilon}\right) .
$$

It remains to choose $R, \varepsilon$ such as to minimize the bound. Taking

$$
R=\left(\frac{\frac{3}{2}\|\boldsymbol{\sigma}\|_{0}}{\|\boldsymbol{\sigma}\|_{L^{\infty}}}\right)^{2 / 3} \quad \text { and } \quad \varepsilon=\left(\frac{4\|\boldsymbol{\sigma}\|_{L^{\infty}}}{\|\boldsymbol{\nabla} \boldsymbol{\sigma}\|_{L^{4}}}\right)^{1 / 4}
$$

we finally obtain

$$
\|\boldsymbol{\nabla} \boldsymbol{u}\|_{L^{\infty}} \leq C\|\boldsymbol{\sigma}\|_{L^{\infty}}\left(1+\log _{+}\|\boldsymbol{\sigma}\|_{0}+\log _{+}\|\boldsymbol{\nabla} \boldsymbol{\sigma}\|_{L^{4}}\right)
$$

where $\log _{+} x=\max (\log x, 0)$.

The estimate (4.7) with $m=0$ gives:

$$
\|\boldsymbol{\sigma}\|_{0} \leq \exp \left[C \int_{0}^{t}\left(1+\|\boldsymbol{\sigma}\|_{L^{\infty}}+\|\boldsymbol{\nabla} \boldsymbol{u}\|_{L^{\infty}}\right) d s\right]\left\|\boldsymbol{\sigma}_{0}\right\|_{0}
$$

and similarly, from (4.8),

$$
\|\boldsymbol{\nabla} \boldsymbol{\sigma}\|_{L^{4}} \leq \exp \left[C \int_{0}^{t}\left(1+\|\boldsymbol{\sigma}\|_{L^{\infty}}+\|\boldsymbol{\nabla} \boldsymbol{u}\|_{L^{\infty}}\right) d s\right]\left\|\boldsymbol{\nabla} \boldsymbol{\sigma}_{0}\right\|_{L^{4}}
$$

Substituting into (4.9) we get

$$
\|\boldsymbol{\nabla} \boldsymbol{u}\|_{L^{\infty}} \leq C\|\boldsymbol{\sigma}\|_{L^{\infty}}\left[1+\int_{0}^{t}\left(1+\|\boldsymbol{\sigma}\|_{L^{\infty}}+\|\boldsymbol{\nabla} \boldsymbol{u}\|_{L^{\infty}}\right) d s\right] .
$$

If we define

$$
M(t)=\int_{0}^{t}\left[1+\|\boldsymbol{\sigma}\|_{L^{\infty}}+\|\boldsymbol{\nabla} \boldsymbol{u}\|_{L^{\infty}}\right] d s \quad \text { and } \quad N(t)=\int_{0}^{t}\|\boldsymbol{\sigma}\|_{L^{\infty}} d s,
$$

then we have an integral inequality of the form

$$
M^{\prime}(t) \leq C N^{\prime}(t)[1+M(t)],
$$

which we readily integrate:

$$
\int_{0}^{t}\left[1+\|\boldsymbol{\sigma}\|_{L^{\infty}}+\|\boldsymbol{\nabla} \boldsymbol{u}\|_{L^{\infty}}\right] d s \leq \exp \left(C \int_{0}^{t}\|\boldsymbol{\sigma}\|_{L^{\infty}} d s\right) .
$$


Combining with (4.7) we have proved Theorem 4.1.

Note that (4.7) together with (4.10) yield a doubly-exponential bound,

$$
\|\boldsymbol{\sigma}\|_{m} \leq \exp \left[C \exp \left(C \int_{0}^{t}\|\boldsymbol{\sigma}\|_{L^{\infty}} d s\right)\right]\left\|\boldsymbol{\sigma}_{0}\right\|_{m} .
$$

In this context, it is noteworthy that a similar doubly-exponential bound was derived in BKM for the Euler equation. Ponce in [22] derives a singly-exponential bound using as control parameter the $L^{\infty}$ norm of the deformation tensor, rather than the vorticity as in the original BKM paper.

\section{Discussion}

In this paper we derived a breakdown condition for solutions of the Oldroyd-B model in $\mathbb{R}^{3}$ in the limit of zero Reynolds number. This condition is analogous to the breakdown condition of Beale-Kato-Majda for Newtonian fluids. It is noteworthy that the elastic relaxation time, $\lambda$ (which in non-dimensional formulations is the Weissenberg number), plays no role in our analysis. In fact, nothing changes if we set $\lambda=\infty$, or, alternatively, if we set $\lambda=\infty$ but retain the ratio $\nu_{p} \lambda$ constant (which corresponds to the Kelvin-Voigt model for a viscoelastic solid).

The main implication of our result is that the efforts toward a global-in-time well-posedness theory should focus on the control of the $L^{\infty}$ norm of the stress.

In a more physically realistic setting, one should consider the same problem in a bounded domain $\Omega$. Let us assume for simplicity homogeneous Dirichlet boundary conditions for $\boldsymbol{u}$, and a sufficiently smooth boundary $\partial \Omega$. The Calderoń-Zygmund inequality holds in this case (see e.g. [23, 15]), so that local-in-time existence can be proved as for the infinite domain. Differences arise in the proof of the BKM criterion, where we need an $L^{\infty}$ estimate for $\boldsymbol{\nabla} \boldsymbol{u}$. For a bounded domain, the integral representation (2.6) can be rewritten with the same kernel integrated over $\Omega$, plus a boundary-term contribution, exactly as in the Poisson representation formula (see [15]). Another alternative is to use the following Green representation formula,

$$
\boldsymbol{\nabla} \boldsymbol{u}(\boldsymbol{x})=\int_{\Omega} \mathcal{G}_{\Omega}(\boldsymbol{y}) \cdot \operatorname{div} \sigma(\boldsymbol{x}-\boldsymbol{y}) d \boldsymbol{y},
$$

where $\mathcal{G}_{\Omega}$ is a singular kernel that depends on $\Omega$. The existence of $\mathcal{G}_{\Omega}$ along with pointwise estimates has to be proved using elliptic regularity theory (see Ferrari [24] for details).

Appendix A. Some inequalities. In this appendix we list a number of inequalities used repeatedly in Secs. 3 and 4. We recall that $H^{m}\left(\mathbb{R}^{3}\right)$ denotes the Sobolev spaces of scalar, vector and multi-dimensional tensor fields, with the corresponding norm $\|\cdot\|_{m}$. The $L^{2}$-norm, which coincides with the $H^{0}$-norm, is denoted by $\|\cdot\|_{0}$. The $L^{\infty}$ norm is denoted by $\|\cdot\|_{L^{\infty}}$. Weak derivatives are denoted by $D^{\alpha}$, where $\alpha=\left(\alpha_{1}, \alpha_{2}, \alpha_{3}\right)$ is a multi-index. by

Mollifiers. For $f \in L^{p}\left(\mathbb{R}^{3}\right), 1 \leq p \leq \infty$, the mollification operator, $J_{\varepsilon}$, is defined

$$
\left(J_{\varepsilon} f\right)(\boldsymbol{x})=\frac{1}{\varepsilon^{3}} \int_{\mathbb{R}^{3}} \phi\left(\frac{\boldsymbol{x}-\boldsymbol{y}}{\varepsilon}\right) f(\boldsymbol{y}) d \boldsymbol{y},
$$

where $\phi: \mathbb{R}^{3} \rightarrow \mathbb{R}$ is a radially symmetric, positive, compactly supported $C^{\infty}$ function, satisfying $\int_{\mathbb{R}^{3}} \phi(\boldsymbol{x}) d \boldsymbol{x}=1$. The important properties satisfied by $J_{\varepsilon}$ are: 
- $J_{\varepsilon}$ commutes with (distributional) derivatives.

- $J_{\varepsilon}$ is symmetric with respect to the $L^{2}\left(\mathbb{R}^{3}\right)$ inner product.

- $J_{\varepsilon}: H^{m}\left(\mathbb{R}^{3}\right) \rightarrow H^{m}\left(\mathbb{R}^{3}\right) \cap C^{\infty}\left(\mathbb{R}^{3}\right)$.

- There exists a $C>0$ such that for every $f \in H^{m}\left(\mathbb{R}^{3}\right)$,

$$
\left\|J_{\varepsilon} f-f\right\|_{m-1} \leq C \varepsilon\|f\|_{m}
$$

- For every $f \in H^{m}\left(\mathbb{R}^{3}\right)$ and $k \geq 0$,

$$
\left\|J_{\varepsilon} f\right\|_{m+k} \leq \frac{c_{m k}}{\varepsilon^{k}}\|f\|_{m} .
$$

- For every $f \in H^{m}\left(\mathbb{R}^{3}\right)$ and multi-index $\alpha$,

$$
\left\|J_{\varepsilon} D^{\alpha} f\right\|_{L^{\infty}} \leq \frac{c_{|\alpha|}}{\varepsilon^{3 / 2+|\alpha|}}\|f\|_{0} .
$$

Banach algebra property of Sobolev spaces. For $m>3 / 2$ the Sobolev space $H^{m}\left(\mathbb{R}^{3}\right)$ is a Banach algebra, i.e., there exists a constant $C>0$ such that for all $f, g \in H^{m}\left(\mathbb{R}^{3}\right)$,

$$
\|f g\|_{m} \leq C\|f\|_{m}\|g\|_{m}
$$

Sobolev calculus inequalities. For all $m \in\{0,1,2, \ldots\}$ there exists a constant $C>0$ such that for all $f, g \in L^{\infty}\left(\mathbb{R}^{3}\right) \cap H^{m}\left(\mathbb{R}^{3}\right)$,

$$
\|f g\|_{m} \leq C\left(\|f\|_{L^{\infty}}\|g\|_{m}+\|f\|_{m}\|g\|_{L^{\infty}}\right) .
$$

For $m>3 / 2$ the Sobolev embedding $H^{m}\left(\mathbb{R}^{3}\right) \subset L^{\infty}\left(\mathbb{R}^{3}\right)$ implies that this inequality is valid for all $f, g \in H^{m}\left(\mathbb{R}^{3}\right)$. Moreover, for $f \in H^{m}\left(\mathbb{R}^{3}\right), g \in H^{m-1}\left(\mathbb{R}^{n}\right)$, and $|\alpha| \leq m$,

$$
\left\|D^{\alpha}(f g)-f D^{\alpha} g\right\|_{0} \leq C\left[\|\nabla f\|_{L^{\infty}}\|g\|_{m-1}+\|f\|_{m}\|g\|_{L^{\infty}}\right] .
$$

This inequality holds even if each of the two subtracted terms on the left hand side is not in $L^{2}\left(\mathbb{R}^{3}\right)[6]$. is

Gagliardo-Nirenberg inequalities. The classical Gagliardo-Nirenberg inequality

$$
\left\|D^{k} f\right\|_{L^{q}} \leq C\|f\|_{L^{r}}^{1-\theta}\left\|D^{m} f\right\|_{L^{p}}^{\theta}
$$

where $\theta=k / m \in(0,1), 1 \leq p, r \leq \infty$, and

$$
\frac{1}{q}=\frac{\theta}{p}+\frac{1-\theta}{r}
$$

For the particular case $r=\infty$, we have

$$
\left\|D^{k} f\right\|_{L^{q}} \leq C\|f\|_{L^{\infty}}^{1-k / m}\left\|D^{m} f\right\|_{L^{k q / m}}^{k / m},
$$

where $1<p<\infty$ and $1 \leq m \leq k$. 
Sobolev embedding theorems ([25] Ch. 5, [26] p. 168). Let $m \geq 1$ be an integer and $1 \leq p<\infty$. Then:

$$
\begin{aligned}
& \text { If } \frac{1}{p}-\frac{m}{n}>0 \text { then } W^{m, p}\left(\mathbb{R}^{n}\right) \subset L^{q}\left(\mathbb{R}^{n}\right) \text { with } \frac{1}{q}=\frac{1}{p}-\frac{m}{n} \\
& \text { If } \frac{1}{p}-\frac{m}{n}=0 \text { then } W^{m, p}\left(\mathbb{R}^{n}\right) \subset L^{q}\left(\mathbb{R}^{n}\right), \forall q \in[p,+\infty) \\
& \text { If } \frac{1}{p}-\frac{m}{n}<0 \text { then } W^{m, p}\left(\mathbb{R}^{n}\right) \subset L^{\infty}\left(\mathbb{R}^{n}\right) .
\end{aligned}
$$

In this paper we use extensively the third embedding for $p=2$ and $n=3$, i.e.,

$$
H^{m}\left(\mathbb{R}^{3}\right) \subset L^{\infty}\left(\mathbb{R}^{3}\right) \quad \text { if } \quad m>\frac{3}{2} .
$$

In fact, this is a continuous embedding, i.e.,

$$
\|f\|_{L^{\infty}} \leq C\|f\|_{m}
$$

The Calderón-Zygmund inequality. Let $K: \mathbb{R}^{3} \rightarrow \mathbb{R}$ be a homogeneous function of degree -3 that averages to zero on the unit sphere, and for $f \in L^{q}\left(\mathbb{R}^{3}\right), q \geq 2$, define

$$
g(\boldsymbol{x})=(\mathrm{P} . \mathrm{V} .) \int_{\mathbb{R}^{3}} K(\boldsymbol{y}) f(\boldsymbol{x}-\boldsymbol{y}) d \boldsymbol{y} .
$$

Then $g \in L^{q}\left(\mathbb{R}^{3}\right)$, and

$$
\|g\|_{L^{q}} \leq C q\|f\|_{L^{q}} .
$$

Because the constant grows unbounded with $q$, this inequality does not carry to the $L^{\infty}$-norm; see, for example, Stein [27] for a proof. In particular, since the same kernel relates $D^{\alpha} f$ and $D^{\alpha} g$, it follows that $f \in H^{m}\left(\mathbb{R}^{3}\right)$ implies $g \in H^{m}\left(\mathbb{R}^{3}\right)$ with

$$
\|g\|_{m} \leq C\|f\|_{m}
$$

Acknowledgments. RK and CM were partially supported by the Israel Science Foundation. The work of EST was supported in part by the NSF grant no. DMS0504619, the ISF grant no. 120/6, and the BSF grant no. 2004271.

\section{REFERENCES}

[1] R.B. Bird, R.C. Armstrong and O. Hassager, Dynamics of Polymeric Liquids, John Wiley and Sons, New York, 1, 1987.

[2] P. Constantin, A.J. Majda and E. Tabak, Formation of strong fronts in the 2-D quasigeostrophic thermal active scalar, Nonlinearity, 7, 1495-1533, 1994.

[3] R. Keunings, On the high Weissenberg number problem, J. Non-Newton. Fluid Mech., 20, 209-226, 1986.

[4] R. Keunings, A survey of computational rheology, in D.M. Binding et al. (editor), 13th Int. Congr. on Rheology, British Society of Rheology, 1, 2000.

[5] R. Fattal and R. Kupferman, Time-dependent simulation of viscoelastic flows at high Weissenberg number using the log-conformation representation, J. Non-Newton. Fluid Mech., 126, 23-37, 2005.

[6] J.T. Beale, T. Kato and A.J. Majda, Remarks on the breakdown of smooth solutions for the 3-D Euler equations, Comm. Math. Phys., 94, 61-66, 1984.

[7] P. Constantin, C. Fefferman and A.J. Majda, Geometric constraints on potentially singular solutions for the 3D Euler equations, Comm. Part. Diff. Eq., 21, 559-571, 1996.

[8] C. Bardos and E.S. Titi, Euler equations of incompressible ideal fluids, Russian Math. Surveys, 2007.

[9] P. Constantin, On the Euler equations of incompressible fluids, (in press), 2007. 
[10] J.Y. Chemin and N. Masmoudi, About lifespan of regular solutions of equations related to viscoelastic fluids, SIAM J. Math. Anal., 33, 84-112, 2001.

[11] F.H. Lin, C. Liu and P. Zhang, On hydrodynamics of viscoelastic fluids, Comm. Pure Appl. Math., LVIII, 1437-1471, 2005.

[12] P. Constantin, Nonlinear Fokker-Planck Navier-Stokes systems, Commun. Math. Sci., 3, 531$544,2005$.

[13] P. Constantin, C. Fefferman, E.S. Titi and A. Zarnescu, Regularity of coupled two-dimensional nonlinear Fokker-Planck and Navier-Stokes systems, Comm. Math. Phys., 270, 789-812, 2007.

[14] P. Constantin and N. Masmoudi, Global well-posedness for a Smoluchowski equation coupled with Navier-Stokes equations in 2D, Comm. Math. Phys., (in press), 2007.

[15] G. Galdi, An Introduction to the Mathematical Theory of the Navier-Stokes Equations, Springer-Verlag, 1, 1994.

[16] A.J. Majda and A.L. Bertozzi, Vorticity and Incompressible Flow, Cambridge University Press, Cambridge, 2002.

[17] T. Li, H. Zhang and P. Zhang, Local existence for the dumbbell model of polymeric fluids, Comm. Part. Diff. Eq., 29, 903-923, 2004.

[18] T. Sideris and B. Thomases, Global existence for $3 D$ incompressible and isotropic elastodynamics via the incompressible limit, Comm. Pure Appl. Math., 58, 750-788, 2005.

[19] H. Zhang and P. Zhang, Local existence for the FENE dumbbell model of polymeric fluids, Arch. Rat. Mech. Anal., 181, 373-400, 2006.

[20] T. Sideris and B. Thomases, Global existence for 3D incompressible isotropic elastodynamics, Comm. Pure Appl. Math., to appear.

[21] Z. Lei, C. Liu and Y. Zhou, Global solutions for incompressible viscoelastic fluids, Arch. Rat. mech. Anal., (in press), 2007.

[22] G. Ponce, Remarks on a paper by J.T. Beale, T. Kato and A. Majda, Comm. Math. Phys., 98, 349-353, 1985.

[23] V.I. Yudovich, Non-stationary flow of an ideal incompressible liquid, Zh. Vych. Mat., 3, 10321066, 1963.

[24] A.B. Ferrari, On the blow-up of solutions of the 3-D Euler equations in a bounded domain, Comm. Math. Phys., 155, 277-294, 1993.

[25] R.A. Adams, Sobolev Spaces, Academic Press, London, 1975.

[26] H. Brezis, Analyse Fonctionnelle, Théorie et Applications, Masson, Paris, 1983.

[27] E.M. Stein, Singular Integrals and Differentiability Properties of Functions, Princeton University Press, Princeton NJ, 1970. 